\title{
NONLINEAR METHODS FOR MODEL REDUCTION
}

\author{
Andrea Bonito ${ }^{1}$, Albert Cohen ${ }^{2}$, Ronald DeVore ${ }^{1}$, Diane Guignard ${ }^{1}$, \\ Peter Jantsch ${ }^{1, *}$ and Guergana Petrova ${ }^{1}$
}

\begin{abstract}
Typical model reduction methods for parametric partial differential equations construct a linear space $V_{n}$ which approximates well the solution manifold $\mathcal{M}$ consisting of all solutions $u(y)$ with $y$ the vector of parameters. In many problems of numerical computation, nonlinear methods such as adaptive approximation, $n$-term approximation, and certain tree-based methods may provide improved numerical efficiency over linear methods. Nonlinear model reduction methods replace the linear space $V_{n}$ by a nonlinear space $\Sigma_{n}$. Little is known in terms of their performance guarantees, and most existing numerical experiments use a parameter dimension of at most two. In this work, we make a step towards a more cohesive theory for nonlinear model reduction. Framing these methods in the general setting of library approximation, we give a first comparison of their performance with the performance of standard linear approximation for any compact set. We then study these methods for solution manifolds of parametrized elliptic PDEs. We study a specific example of library approximation where the parameter domain is split into a finite number $N$ of rectangular cells, with affine spaces of dimension $m$ assigned to each cell, and give performance guarantees with respect to accuracy of approximation versus $m$ and $N$.
\end{abstract}

Mathematics Subject Classification. 41A10, 41A58, 41A63, 65N15.

Received May 11, 2020. Accepted August 3, 2020.

\section{INTRODUCTION}

Complex systems are frequently described by parametric partial differential equations (PDEs) that take the general form

$$
\mathcal{P}(u, y)=0,
$$

where $y$ ranges over some parameter domain $Y$, and $u=u(y)$ is the corresponding solution which is assumed to be uniquely defined in some Hilbert space $V$ for every $y \in Y$. We denote by $\|\cdot\|=\|\cdot\|_{V}$ and $\langle\cdot, \cdot\rangle$ the norm and inner product of $V$, respectively. In what follows, we assume that the parameters are countably infinite and have been rescaled so that $Y=[-1,1]^{\mathbb{N}}$. The case of a finite dimensional parameter $y=\left(y_{1}, \ldots, y_{J}\right)$ can always be recast in this setting by considering that $u(y)$ does not depend of the variable $y_{j}$ for $j>J$.

There are three main problem areas associated with parametric PDEs:

Keywords and phrases. Parametric PDEs, reduced modeling, piecewise polynomials.

1 Department of Mathematics, Texas A\&M University, College Station, TX 77840, USA.

2 Laboratoire Jacques-Louis Lions, Sorbonne Université, Paris, France.

* Corresponding author: pjantsch@tamu.edu 
(i) building forward solvers to efficiently compute approximations to $u(y)$ for any given $y \in Y$;

(ii) estimating the state $u(y)$ from data observation when the parameter $y$ is unknown;

(iii) estimating the parameter $y$ that can give rise to an observed state $u$.

One commonly used approach to tackle these three ranges of problems in a numerically efficient way is reduced modeling. In its most usual form, it is based on introducing a linear space $V_{n}$ of low dimension $n$ which is tailored to provide an accurate approximation to all solutions $u(y)$ as $y$ varies in $Y$, or equivalently, to the solution manifold,

$$
\mathcal{M}:=\{u(y): y \in Y\}
$$

\subsection{Linear reduced models}

There are two common approaches to finding a reduced model $V_{n}$. The first one is to establish that the forward map $y \mapsto u(y)$ has a certain analyticity in $y$, and thereby admits a Taylor series representation

$$
u(y)=\sum_{\nu \in \mathcal{F}} t_{\nu} y^{\nu}, \quad t_{\nu} \in V .
$$

Here $\mathcal{F}$ denotes the set of $\nu=\left(\nu_{1}, \nu_{2}, \ldots\right)$ which have finite support and whose entries are nonnegative integers. Quantitative bounds for the size of the Taylor coefficients $t_{\nu}$ allow one to prove that for each $\varepsilon$, there is a finite set $\Lambda=\Lambda(\varepsilon) \subset \mathcal{F}$ such that

$$
\sup _{y \in Y}\left\|u(y)-\sum_{\nu \in \Lambda} t_{\nu} y^{\nu}\right\|_{V} \leq \varepsilon .
$$

The space $V_{n}:=\operatorname{span}\left\{t_{\nu}: \nu \in \Lambda\right\}$ provides the reduced model with $n=\#(\Lambda)$. In this case, an approximation of $u(y)$ in $V_{n}$ is readily provided by the function

$$
\hat{u}(y):=\sum_{\nu \in \Lambda} t_{\nu} y^{\nu}
$$

that is, using the $y^{\nu}$ as the coefficients of $\hat{u}$ in the basis $t_{\nu}$. Quantitative bounds on the cardinality of $\Lambda(\varepsilon)$ are known under various assumptions on the coefficients of the PDE [6].

The second approach to finding a reduced model is to judiciously select certain snapshots $u\left(y^{1}\right), \ldots, u\left(y^{n}\right)$ of $u$ via a greedy procedure, and use the space $V_{n}:=\operatorname{span}\left\{u\left(y^{1}\right), \ldots, u\left(y^{n}\right)\right\}$ as the reduced model. In this case, the approximation of $u(y)$ in $V_{n}$ requires a projection step.

Recent results show that there can be a numerical advantage to finding a reduced basis by the Taylor coefficient approach. In the case of elliptic and certain parabolic PDEs, it is sometimes possible to find the set $\Lambda$ a priori by exploiting the parametric form of the diffusion coefficients [1]. This avoids computationally expensive search algorithms that are a component of greedy reduced basis selections. On the other hand, greedy procedures have the advantage that they are provably near-optimal for finding a linear space to approximate $u$, in the sense that their convergence rates are similar to those of the optimal linear spaces for approximating $\mathcal{M}$ [2]. Moreover, as we illustrate further in this paper, numerical experiments show that for a prescribed target accuracy, the greedy generated spaces that meet this accuracy are of significantly lower dimension than their polynomial counterparts.

There is a rigorous theory that quantifies the approximation performance of both of these reduced models; see [6] for a summary of known results. The theory is most fully developed in the case of elliptic PDEs of the form

$$
-\operatorname{div}(a \nabla u)=f,
$$

set on a physical domain $D \subset \mathbb{R}^{d}$, say with Dirichlet boundary conditions $u_{\mid \partial D}=0$, and where the diffusion function $a$ has an affine parametrization

$$
a(y)=\bar{a}+\sum_{j \geq 1} y_{j} \psi_{j},
$$


for some given functions $\bar{a}$ and $\left(\psi_{j}\right)_{j \geq 1}$ in $L^{\infty}(D)$. These functions are assumed to satisfy the condition

$$
\left\|\frac{\sum_{j \geq 1}\left|\psi_{j}\right|}{\bar{a}}\right\|_{L^{\infty}(D)}<1,
$$

which is equivalent to the following assumption.

Uniform Ellipticity Assumption (UEA): there exist $0<a_{\min } \leq a_{\max }<\infty$ such that

$$
0<a_{\min } \leq a(y) \leq a_{\max }<\infty, \quad y \in Y .
$$

Lax-Milgram theory then ensures that whenever $f \in V^{\prime}=H^{-1}(D)$, for each $y \in Y$, the corresponding solution $u(y)$ is uniquely defined in the Hilbert space $V:=H_{0}^{1}(D)$ endowed with the norm $\|\cdot\|_{V}:=\|\nabla \cdot\|_{L^{2}(D)}$.

\subsection{Nonlinear reduced models}

It is known that in many contexts, numerical methods based on nonlinear approximation outperform linear methods, in the sense of requiring a much reduced computational cost to achieve a prescribed error tolerance [9]. This motivates us to consider replacing the linear space $V_{n}$ by a nonlinear space $\Sigma_{n}$ depending on $n$ parameters. We call such a space $\Sigma_{n}$ a nonlinear reduced model. This idea has already been suggested and studied in certain settings; see e.g. $[11,12,16]$. However, up till now, there has not been a unified study of nonlinear model reduction. The purpose of the present paper is to provide a formal theory for such methods of nonlinear model reduction and to prove some first results that quantify their performance.

The nonlinear reduced models studied in this paper can be placed into the form of what is sometimes called library approximation. Given a Banach space $X$, a library $\mathcal{L}$ is a finite collection of affine spaces $L_{j}:=$ $x_{j}+X_{j}, j=1, \ldots, N$, where each $X_{j}$ is a linear space of dimension at most $m$, and each $x_{j} \in X, j=1, \ldots, N$. We set each $X_{j}=\{0\}$ in the case $m=0$. For an element $x \in X$, the error of approximation of $x$ by the library $\mathcal{L}$ is

$$
E(x, \mathcal{L}):=\inf _{L \in \mathcal{L}} \operatorname{dist}(x, L)_{X} .
$$

In other words, given $x$, we choose the best of the affine spaces $L_{j}=x_{j}+X_{j}, j=1, \ldots, N$, to approximate $x$. Given a library $\mathcal{L}$ and a compact set $K \subset X$, we define

$$
E_{\mathcal{L}}(K):=\sup _{x \in K} E(x, \mathcal{L})
$$

Here, in the context of reduced models for parametric PDEs, the idea is to keep $m$ small when compared to the dimension $n$ used in linear models $V_{n}$, while retaining the same accuracy of the reduced model.

For parametric PDEs, we take $X=V$ and $K=\mathcal{M}:=\{u(y): y \in Y\}$ to be the solution manifold of the PDE. A library $\mathcal{L}$ would then consist of affine spaces

$$
L_{j}:=u_{j}+V_{j},
$$

where each $u_{j} \in V$ and each $V_{j} \subset V$ has dimension at most $m$. Then, the best approximation to $u(y)$ from $L_{j}$ is

$$
u_{j}+P_{V_{j}}\left(u(y)-u_{j}\right)
$$

where $P_{V_{j}}$ is the $V$-orthogonal projection onto $V_{j}$. In this context, when presented with a parameter $y$ for which we wish to compute an online approximation to $u(y)$, the choice of which space $L_{j}$ to use from a given library $\mathcal{L}$ could be decided in several ways, among which we mention:

(i) searching for a computable bound for $\operatorname{dist}\left(u(y), L_{j}\right)_{V}=\left\|u-u_{j}-P_{V_{j}}\left(u(y)-u_{j}\right)\right\|_{V}$, and choosing the value of $j$ that minimizes this surrogate quantity; 
(ii) building an a priori partition of the parameter domain $Y$ into cells $Q_{j}$ and construct an $L_{j}$ for each cell. Then the choice of $L_{j}$ for approximating $u(y)$ is determined by the cell $Q_{j}$ containing $y$.

Only the latter procedure is considered in this paper.

Returning back to the case of a general Banach space $X$, we denote by $\mathrm{E}_{m, N}=\mathrm{E}_{m, N}(X)$ the collection of all libraries $\mathcal{L}=\left\{L_{1}, \ldots, L_{N}\right\}$ containing $N$ affine spaces of dimension at most $m$. If we fix the values of $m$ and $N$, then the best performance of a library with these fixed values is

$$
d_{m, N}(K):=\inf _{\mathcal{L} \in \mathrm{E}_{m, N}} E_{\mathcal{L}}(K)
$$

We call $d_{m, N}$ the library width of $K$. This definition slightly differs from that introduced in [15] in which the spaces $L_{j}$ are taken to be linear instead of affine.

Library widths include the two standard approximation concepts of widths and entropy. Recall that if $K$ is a compact set in a Banach space $X$ then its Kolmogorov $m$ width is

$$
d_{m}(K):=d_{m}(K)_{X}:=\inf _{\operatorname{dim}(Y)=m} \operatorname{dist}(K, Y)_{X},
$$

where the infimum is taken over all linear spaces $Y$ of dimension $m$. Thus the Kolmogorov $m$ width of $K$ is the smallest error that can be obtained by approximation by linear spaces of dimension $m$. It follows that we can sandwich the library width $d_{m, 1}(K)_{X}$ between Kolmogorov widths by

$$
d_{m+1}(K) \leq d_{m}\left(K_{0}\right)=d_{m, 1}(K) \leq d_{m}(K),
$$

where $K_{0}=K-x_{0}$ for some suitable $x_{0} \in X$. At the other extreme,

$$
d_{0,2^{n}}(K)=\varepsilon_{n}(K)
$$

where $\varepsilon_{n}(K)$ is the $n$-th entropy number of $K$, that is, the smallest number $\varepsilon$ such that $K$ can be covered by $2^{n}$ balls in $X$ of radius $\varepsilon$.

The construction of linear reduced models via greedy algorithms has offline cost that increases exponentially as the dimension of the reduced space increases. This is due to the fact that the greedy algorithm needs to search for the reduced basis elements through a large training set, which should in principle resolve the solution manifold $\mathcal{M}$ to the same accuracy $\varepsilon$ that is targeted for the reduced basis space $V_{n}$. This is one of the motivations for using library approximation with a small value of $m$ in the context of parametric PDEs. For example, it is known that if the Kolmogorov $n$ width of the solution manifold $d_{n}(\mathcal{M})$ decays like $\mathcal{O}\left(n^{-s}\right)$ for some $s>0$, then taking $\varepsilon=n^{-s}$, the training set needed to achieve accuracy $\varepsilon$ should have cardinality $\mathcal{O}\left(e^{C \varepsilon^{-1 / s}}\right)$, or equivalently $\mathcal{O}\left(e^{c n}\right)$, for some fixed constants $C, c>0$. The resulting offline cost becomes prohibitive as $\varepsilon$ is getting small (or $n$ is getting large). The reader can find a detailed analysis of this cost of greedy constructions in [6] or [8]. We should note that it was recently shown in [8] that the offline cost of greedy constructions (under certain model assumptions on the parametric coefficients) can be reduced to polynomial growth in $\varepsilon$ by using a random training set, provided we are now willing to accept results that hold with high probability. In order not to confuse various issues, we put this aside when going further in this paper.

Because of the offline cost, it may be impossible to build a linear model using a greedy algorithm when the user prescribed error is too small. On the other hand, by choosing $m$ small and an appropriate partitioning $\left(Q_{j}\right)_{j=1, \ldots, N}$ for $Y$, the offline cost is moderate and a nonlinear reduced model may be constructed provided $N$ is not too large. Keeping $m$ small may also be useful in other contexts, such as reducing the online cost for the forward problem and numerical savings for state and parameter estimation. In fact the latter is one of our main motivations for nonlinear reduced models, and we discuss it in more detail in Section 4. 


\subsection{Outline of the paper}

We begin in Section 2 by giving some general remarks on library approximation for a general compact set $K$ in a Banach space $X$ whose Kolmogorov $n$ widths decay like $\mathcal{O}\left(n^{-r}\right)$ for some $r>0$. Then given any target accuracy $\varepsilon$ and writing $\varepsilon=n^{-r}$ for a suitable integer $n$, we show that $d_{m, N}(K) \leq \varepsilon$ provided $N \geq 2^{c(n-m)}$, with $c$ depending only on $r$. This result gives a bound on how many spaces would be needed in the library if we restrict the dimension of the component spaces $X_{j}$ to be at most $m$. This provides a first quantitative bound for general compact sets. However, it is well known that nonlinear methods are not beneficial for certain compact sets, and thus this estimate is very pessimistic.

From Section 3, our paper is directed at using library approximation for reduced models for parametric PDEs. We take $K=\mathcal{M}$ where $\mathcal{M}$ is the solution manifold of a parametric elliptic PDE with affine coefficients (1.7). As already indicated, the library approximation studied in this paper can be viewed as first partitioning the parameter set into $N$ cells $Q_{j}$ and assigning an affine space $L_{j}=u_{j}+V_{j}$ with $V_{j}$ of dimension at most $m$ on each cell. The main issues therefore are how to choose the cells and how to design the spaces $V_{j}$. Given a target accuracy $\varepsilon$ and a prescribed target $m$ for the dimension of the spaces in the library, we are interested in strategies for generating a good partition of $Y$ into $N$ cells with a bound on the number $N$ of cells needed to guarantee the prescribed accuracy.

In light of these issues, we consider a tensor product strategy for subdividing the parameter domain into hyperrectangles $Q_{j}$, and find a polynomial space of dimension $m+1$ associated with each rectangle. Thus, the reduced model can be viewed as a piecewise polynomial (in $y$ ) approximation to $u(y)$. We then give bounds on $N$ which are a significant improvement over those in Section 2 and show how these results can be used to give concrete bounds when specific assumptions are made on the affine representation (1.7).

In Section 4, we present the results of various numerical tests that confirm our theoretical results. First, we compare the performance (on the entire parameter domain $Y$ ) of the two primary linear reduced models, namely polynomial and greedy. These results show a dramatic gain in using greedy algorithms in our examples. We implement our numerical methods for partitioning in the case of piecewise polynomial nonlinear models, where our examples show that suitable error can be achieved with a reasonable number of cells provided $m$ is not too small. We then provide a discussion and numerical experiments of nonlinear models based on piecewise polynomials in the setting of data assimilation.

Finally in Section 5, we conclude with remarks on the possible advantages and disadvantages of library based reduced models for applications such as online solvers, data assimilation, and parameter estimation. This section also gives us an opportunity to mention several areas where further research is needed for a better understanding of nonlinear model reduction.

\section{General REMARKS ON LiBRARY APPROXimation}

We begin by making some general remarks on library approximation. The central issue we address in this section is the size of the library needed to achieve a given target accuracy when we require dimension $m$ of the spaces in the library. The following theorem gives a first, very pessimistic, bound for the size of the library, which we denote by $N$.

Theorem 2.1. Let $K$ be a compact set in a Banach space $X$. If for some $x_{0} \in X$ the Kolmogorov widths of $K_{0}=K-x_{0}$ satisfy

$$
d_{k}\left(K_{0}\right)_{X} \leq M k^{-r}, \quad k \geq 1,
$$

for some $M>0$, then for any $0 \leq m \leq n$, one has

$$
d_{m, N}(K) \leq\left(1+2^{2 r}\right) M n^{-r},
$$

provided $N \geq B_{r}^{n-m}$ with $B_{r}$ depending only on $r$. In other words, we can obtain the same accuracy as in (2.1) by using spaces of the lower dimension $m$, provided we take $N$ of them. 
Proof. Since $K=K_{0}+x_{0}$ and since the definition $d_{m, N}(K)$ uses libraries of affine spaces, it is sufficient to prove the theorem for $x_{0}=0$ and thus $K_{0}=K$.

Let us first note that there is a nested sequence of spaces $X_{k} \subset X_{k+1}$ with $\operatorname{dim}\left(X_{k}\right)=k$ and

$$
\operatorname{dist}\left(K, X_{k}\right)_{X} \leq 2^{2 r} M k^{-r}, \quad k \geq 1 \text {. }
$$

Indeed, from (2.1), there are linear spaces $L_{j}, j \geq 0$, of dimension $2^{j}$, and

$$
\operatorname{dist}\left(K, L_{j}\right)_{X} \leq M 2^{-j r} .
$$

The spaces $Y_{j}:=L_{0}+\cdots+L_{j}$ have dimension $n_{j}$ with $2^{j} \leq n_{j} \leq 2^{j+1}$, and satisfy

$$
\operatorname{dist}\left(K, Y_{j}\right)_{X} \leq M 2^{-j r}=2^{2 r} M 2^{-(j+2) r} \leq 2^{2 r} M n_{j+1}^{-r}, \quad j \geq 0 .
$$

Since the spaces $Y_{j}$ are nested, and $n_{0} \leq \ldots \leq n_{j} \leq \ldots$, we can find functions $\phi_{1}, \phi_{2}, \ldots$, such that for each $j$, the functions $\phi_{1}, \ldots, \phi_{n_{j}}$ are a basis for $Y_{j}$. The spaces

$$
X_{0}:=\{0\}, \quad X_{k}:=\operatorname{span}\left\{\phi_{1}, \ldots, \phi_{k}\right\}, \quad k \geq 1,
$$

provide such a nested sequence, since for $n_{j} \leq k \leq n_{j+1}$ we have $Y_{j} \subset X_{k} \subset Y_{j+1}$ and

$$
\operatorname{dist}\left(K, X_{k}\right)_{X} \leq \operatorname{dist}\left(K, Y_{j}\right)_{X} \leq 2^{2 r} M n_{j+1}^{-r} \leq 2^{2 r} M k^{-r}, \quad k \geq 1 .
$$

Case 1. We fix $m$ and first consider the case when $n=m+2^{j}$ with $j=-1,0,1, \ldots$, where for the purposes of this proof we replace $2^{-1}$ by 0 when $j=-1$. We proceed by induction on $j$ and use the nested spaces $X_{k}$ defined above. We define $W:=X_{m}$, which is a space of dimension $m$, and for each $j \geq 0$, we further define

$$
Z_{j}:=\operatorname{span}\left\{\phi_{m+1}, \ldots, \phi_{m+2^{j}}\right\}, \quad \operatorname{dim}\left(Z_{j}\right)=2^{j}, \quad \text { and thus } W+Z_{j}=X_{m+2^{j}} .
$$

We show by induction that for each $j \geq-1$, there is a set $S_{j} \subset Z_{j}$ such that:

(i) the library $\mathcal{L}_{j}:=\left\{s+W, s \in S_{j}\right\}$ provides the approximation error

$$
E_{\mathcal{L}_{j}}(K) \leq\left(1+2^{2 r}\right) M\left[m+2^{j}\right]^{-r}, \quad j \geq-1 ;
$$

(ii) for each $j \geq-1$, the cardinality of $S_{j}$ is

$$
\#\left(S_{j}\right)=: N_{j} \leq\left(1+2^{r+1} R\right)^{2^{j+1}}, \quad R:=1+2^{2 r+1} .
$$

When $j=-1$, we can take the set $S_{-1}=\{0\}$. We obtain the desired error bound (i) because of (2.3), and (ii) is clear since $N_{-1}=\#\left(S_{-1}\right)=1$.

Suppose now that we have established (i) and (ii) for some value of $j$. To advance the induction to $j+1$ we do the following. Let $\hat{X}:=X / W$ denote the quotient space of $X$ modulo $W$ with elements $[x]=x+W$, $x \in X$. We equip this space with its usual norm

$$
\|[x]\|_{\hat{X}}:=\operatorname{dist}(x, W)_{X} .
$$

We then have the finite dimensional spaces $\hat{Z}_{j}:=\left\{[z]: z \in Z_{j}\right\}, j=0,1, \ldots$ For each $z_{\ell} \in S_{j} \subset Z_{j}$, we define

$$
B_{\ell}=B\left(\left[z_{\ell}\right], R_{0}\right):=\left\{[z] \in \hat{Z}_{j+1}:\left\|[z]-\left[z_{\ell}\right]\right\|_{\hat{X}} \leq R_{0}\right\}, \quad R_{0}:=R M\left[m+2^{j}\right]^{-r},
$$

the ball in $\hat{Z}_{j+1}$ with center $\left[z_{\ell}\right]$ and radius $R_{0}$. It is known [14, p.63] that for any $\varepsilon>0$, the covering number $N_{\varepsilon}(U)$ for the unit ball $U$ in $\hat{Z}_{j+1}$ satisfies

$$
N_{\varepsilon}(U) \leq(1+2 / \varepsilon)^{2^{j+1}}
$$


We next set $\varepsilon:=M\left[m+2^{j+1}\right]^{-r}$. It follows that the covering number of $B_{\ell}$ satisfies

$$
N_{\varepsilon}\left(B_{\ell}\right) \leq\left(1+2 R M\left[m+2^{j}\right]^{-r} / \varepsilon\right)^{2^{j+1}} \leq\left(1+2^{r+1} R\right)^{2^{j+1}}, \quad \ell=1, \ldots, N_{j} .
$$

Consider now the union of all the $\varepsilon$ coverings of the balls $B_{\ell}, \ell=1, \ldots, N_{j}$, and define $S_{j+1} \subset Z_{j+1}$ to be the collection $\{s\}$ of representatives of the centers $[s]$ of these balls, that is

$$
\bigcup_{\ell=1}^{N_{j}} B_{\ell} \subset \bigcup_{s \in S_{j+1}} B([s], \varepsilon) .
$$

Clearly,

$$
\#\left(S_{j+1}\right) \leq N_{j}\left(1+2^{r+1} R\right)^{2^{j+1}} \leq\left(1+2^{r+1} R\right)^{2^{j+2}},
$$

where we have used the induction hypothesis (ii) in the first inequality. This advances the induction assumption for the bound on \# $\left(S_{j}\right)$.

We now check that the library $\mathcal{L}_{j+1}:=\left\{s+W, s \in S_{j+1}\right\}$ provides the desired approximation error bound. Let $x \in K$. Then, it follows from (2.3) that there is a $z \in Z_{j+1}$ such that

$$
\|[x]-[z]\|_{\hat{X}} \leq 2^{2 r} M\left[m+2^{j+1}\right]^{-r} .
$$

We also know from our induction hypothesis (i) that there is a $z_{\ell} \in S_{j}$, such that

$$
\left\|[x]-\left[z_{\ell}\right]\right\|_{\hat{X}} \leq\left(1+2^{2 r}\right) M\left[m+2^{j}\right]^{-r} .
$$

Hence,

$$
\left\|[z]-\left[z_{\ell}\right]\right\|_{\hat{X}} \leq\|[x]-[z]\|_{\hat{X}}+\left\|[x]-\left[z_{\ell}\right]\right\|_{\hat{X}} \leq\left(1+2^{2 r+1}\right) M\left[m+2^{j}\right]^{-r},
$$

and so $[z]$ is in the ball $B_{\ell}$. Therefore, there is an $s \in S_{j+1}$ such that

$$
\|[z]-[s]\|_{\hat{X}} \leq M\left[m+2^{j+1}\right]^{-r} .
$$

Combining this with (2.10), we obtain

$$
\|[x]-[s]\|_{\hat{X}} \leq\left(1+2^{2 r}\right) M\left[m+2^{j+1}\right]^{-r} .
$$

This advances our induction hypothesis on the error bound.

Case 2. We consider any $n$, not necessarily of the form $m+2^{j}$. For any $j$ such that $m+2^{j} \geq n$, the library $\mathcal{L}_{j}$ will provide the error $\left(1+2^{2 r}\right) M n^{-r}$ because of $(2.5)$. So, we choose $j$ as the smallest integer such that $2^{j} \geq n-m$. For this value of $j$, we have $2^{j-1} \leq n-m$, and from (2.6) we obtain the bound

$$
N_{j} \leq\left(1+2^{r+1} R\right)^{2^{j+1}}=B_{r}^{2^{j-1}} \leq B_{r}^{n-m},
$$

with $B_{r}:=\left(1+2^{r+1} R\right)^{4}$.

Remark 2.2. We may restate Theorem 2.1 as follows. If

$$
d_{k}\left(K_{0}\right) \leq M k^{-r}, \quad k \geq 1,
$$

then for any $\varepsilon>0$ and $m \geq 0$, there exists a library $\mathcal{L}$ of $m$ dimensional affine spaces, which approximates $K$ to accuracy $\varepsilon$ and has cardinality

$$
N=\#(\mathcal{L}) \leq \exp \left(\alpha \varepsilon^{-1 / r}-\beta m\right),
$$

with $\beta=\ln \left(B_{r}\right)$ and $\alpha=\ln \left(B_{r}\right)\left[M\left(1+2^{2 r}\right)\right]^{1 / r}$. In particular, the library widths of $K$ satisfy

$$
d_{m, N}(K) \leq \varepsilon, \quad \text { whenever } \quad N \geq \exp \left(\alpha \varepsilon^{-1 / r}-\beta m\right) .
$$


Theorem 2.1 is very pessimistic since it holds for all compact sets $K$ and general Banach spaces $X$. As we know in other settings, some compact model classes do not benefit from nonlinear approximation. Also, note that in the proof of the theorem, we use the same space $W$ of dimension $m$ for each of the affine spaces $L_{j}$, thereby never taking advantage of any local behavior of the set $K$. In the following sections of this paper, we study library approximation for the purpose of creating a nonlinear model reduction for parametric elliptic PDEs. We exploit known theorems on the smoothness of the mapping $y \mapsto u(y)$ to give explicit non-uniform and anisotropic tensor product partitions of the parameter domain $Y$ into $N$ cells, and create a library of affine spaces that achieves a prescribed target error and whose size obeys much better bounds than those given in this section.

\section{Piecewise polynomial approximation for parametric PDEs}

Before beginning our analysis, we first remark on what we can expect as quantitative results. Nonlinear methods are most effective when the target function, in our case $u$, is not smooth; for example when it has point singularities or singularities on lower dimensional sets, or it is piecewise smooth. For the parameter to solution map $y \mapsto u(y)$ associated to the elliptic equation (1.6) with affine parametrization (1.7), singularities occur when the function $a(y)$ is not strictly positive. The uniform ellipticity assumption (1.9) ensures that the singularities of $u$ are located outside the parameter domain $Y$. However, as $a_{\min } / a_{\max }$ becomes small, they get closer to the boundary of $Y$, and the use of nonlinear methods becomes more relevant in those cases.

We shall see that the necessary number of cells for a partition generated by the nonlinear method remains modest when a reasonable number of terms $m$ in the polynomial approximation are used on each cell; see Table 1. In the final section of this paper, we discuss the advantages this fact provides for online solvers and problems of state estimation.

\subsection{Polynomial approximation error}

If $\Lambda \subset \mathcal{F}$ is a finite set of indices, we denote by $\mathcal{P}_{\Lambda}$ the linear space of all $V$ valued polynomials

$$
P(y)=\sum_{\nu \in \Lambda} c_{\nu} y^{\nu}
$$

where the coefficients $c_{\nu}$ are in $V$. Here and later we use standard multivariate notation, for example, $y^{\nu}=y_{1}^{\nu_{1}} \cdots$ when $\nu$ has finite support. We always assume that the set $\Lambda$ is a downward closed (or lower) set, that is,

$$
\nu \in \Lambda \text { and } \mu \leq \nu \Longrightarrow \mu \in \Lambda,
$$

where $\mu \leq \nu$ means that $\mu_{j} \leq \nu_{j}$ for all $j$. In particular, the null multi-index is contained in $\Lambda$. Once the coefficients $c_{\nu}$ are fixed, each $P(y)$ is in the affine space

$$
c_{0}+\operatorname{span}\left\{c_{\nu} \in V: \nu \in \Lambda^{*}\right\}, \quad \Lambda^{*}:=\Lambda \backslash\{0\},
$$

which has dimension no more than $\#\left(\Lambda^{*}\right)=\#(\Lambda)-1$. A typical choice for the $c_{\nu}$ are the Taylor coefficients in the expansion (1.3).

When proving results on polynomial approximation to $u$, there are two types of commonly employed assumptions on the diffusion coefficient. The first type of assumption is to assume a decay rate for the sequence $\left(\left\|\psi_{j}\right\|_{L_{\infty}(D)}\right)_{j \geq 1}$. The second is to assume a local interaction bound on how the supports of the $\psi_{j}$ overlap. This latter type is described in [1], and is the assumption we employ here. One could derive bounds similar to those given below in the first setting as well.

We assume throughout this section that $u(y)$ is the solution to (1.6) with diffusion coefficient $a(y)$ given by (1.7) and that there is a positive sequence $\left(\rho_{j}\right)_{j \geq 1}$ such that

$$
\kappa:=\min _{j \geq 1} \rho_{j}>1
$$


and

$$
\delta:=\left\|\frac{\sum_{j \geq 1} \rho_{j}\left|\psi_{j}\right|}{\bar{a}}\right\|_{L^{\infty}(D)}<1 .
$$

The following theorem gives a bound for the error of approximation of $u$ by polynomials from $P_{\Lambda}$.

Theorem 3.1. Assume that (3.4) and (3.5) hold with $\left(\rho_{j}^{-1}\right)_{j \geq 1} \in \ell_{q}(\mathbb{N})$ for $0<q<2$. For each $m \geq 1$, there is a set $\Lambda$ with $\#(\Lambda)=m$ such that the $V$ valued polynomial $P(y):=\sum_{\nu \in \Lambda} t_{\nu} y^{\nu}, y \in Y$, satisfies

$$
\sup _{y \in Y}\|u(y)-P(y)\|_{V} \leq C(\delta, \rho, q)\left\|\left(\rho_{j}^{-1}\right)_{j \geq 1}\right\|_{\ell_{q}} m^{-r}, \quad r=1 / q-1 / 2,
$$

where $C(\delta, \rho, q):=C(\rho, q) C_{\delta}$ with

$$
C(\rho, q):=\beta^{\frac{1}{q}} \exp \left(\frac{\beta}{q}\left\|\left(\rho_{j}^{-1}\right)_{j \geq 1}\right\|_{\ell_{q}}^{q}\right), \quad \beta:=-\ln \left(1-\kappa^{-q}\right) \kappa^{q}, \quad C_{\delta}^{2}:=\frac{(2-\delta) a_{\max }}{(2-2 \delta) a_{\min }^{3}}\|f\|_{V^{\prime}}^{2} .
$$

The set $\Lambda$ can be chosen to be a lower set and is derived explicitly in the proof.

Proof. The proof follows from a general summability result established in [1] together with concrete estimates for the constants given in [4]. For the completeness and clarity of the present paper, we provide the details. We first choose $\Lambda$ to be the set of indices in $\mathcal{F}$ that correspond to the $m$ largest of the numbers $\rho^{-\nu}$. Ties are handled in such a way that $\Lambda$ is a lower set, see [4]. Then, for $P(y):=\sum_{\nu \in \Lambda} t_{\nu} y^{\nu}$ we have by Hölder's inequality that for any $y \in Y$,

$$
\|u(y)-P(y)\|_{V} \leq \sum_{\nu \notin \Lambda}\left\|t_{\nu}\right\|_{V} \leq\left(\sum_{\nu \in \mathcal{F}} \rho^{2 \nu}\left\|t_{\nu}\right\|_{V}^{2}\right)^{\frac{1}{2}}\left(\sum_{\nu \notin \Lambda} \rho^{-2 \nu}\right)^{\frac{1}{2}}
$$

From the proof of Theorem 2.2 in [1], we know also that

$$
\sum_{\nu \in \mathcal{F}} \rho^{2 \nu}\left\|t_{\nu}\right\|_{V}^{2} \leq \frac{(2-\delta)\|\bar{a}\|_{L^{\infty}(D)}}{(2-2 \delta) \inf _{x \in D} \bar{a}(x)^{3}}\|f\|_{V^{\prime}}^{2} \leq C_{\delta}^{2},
$$

where $C_{\delta}$ is defined in (3.7).

Moreover, we have

$$
\sum_{\nu \notin \Lambda} \rho^{-2 \nu}=\sum_{\nu \notin \Lambda} \rho^{-\nu(2-q)} \rho^{-\nu q} \leq\left(\sup _{\nu \notin \Lambda} \rho^{-\nu(2-q)}\right) \sum_{\nu \notin \Lambda} \rho^{-\nu q} .
$$

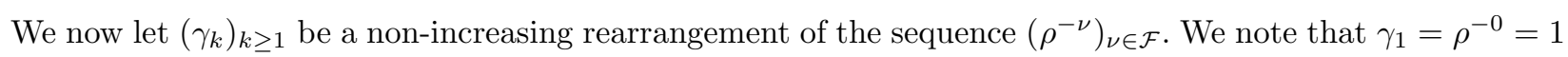
due to the fact that $\rho_{1}>1$ and $\left(\rho_{j}\right)_{j \geq 1}$ is non-decreasing. Then we have

$$
\sup _{\nu \notin \Lambda} \rho^{-\nu q}=\gamma_{m+1}^{q} \leq m^{-1} \sum_{k=2}^{m+1} \gamma_{k}^{q} \leq m^{-1} \sum_{k \geq 2} \gamma_{k}^{q}=m^{-1} \sum_{\nu \neq 0} \rho^{-q \nu}
$$

and hence

$$
\sup _{\nu \notin \Lambda} \rho^{-\nu(2-q)} \leq\left(m^{-1} \sum_{\nu \neq 0} \rho^{-q \nu}\right)^{\frac{2-q}{q}}
$$


Using (3.9) and (3.12) with (3.10) in (3.8), we get

$$
\begin{aligned}
\|u(y)-P(y)\|_{V} & \leq C_{\delta}\left(m^{-1} \sum_{\nu \neq 0} \rho^{-q \nu}\right)^{\frac{2-q}{2 q}}\left(\sum_{\nu \notin \Lambda} \rho^{-\nu q}\right)^{\frac{1}{2}} \\
& \leq C_{\delta} m^{-\frac{1}{q}+\frac{1}{2}}\left(\sum_{\nu \neq 0} \rho^{-\nu q}\right)^{\frac{1}{q}} .
\end{aligned}
$$

The final step of the proof is giving an upper bound of the term $\sum_{\nu \neq 0} \rho^{-q \nu}$. For this, let $\alpha:=\kappa^{-q}<1$, so that $\rho_{j}^{-q} \leq \alpha$ for all $j \geq 1$. Now define $\beta \geq 1$ so that $1-\alpha=e^{-\beta \alpha}$, i.e., $\beta$ is the same as defined in (3.7). Then, $\beta$ depends only on $\kappa$, and $q$, and by the convexity of $e^{-\beta x}$, we have $1-x \geq e^{-\beta x}$ for $0 \leq x \leq \alpha$. It follows that $\left(1-\rho_{j}^{-q}\right)^{-1} \leq e^{\beta \rho_{j}^{-q}}$, and therefore

$$
\sum_{\nu \neq 0} \rho^{-q \nu}=\prod_{j=1}^{\infty}\left(1-\rho_{j}^{-q}\right)^{-1}-1 \leq e^{\beta b}-1 \leq \beta b e^{\beta b}, \quad b:=\left\|\left(\rho_{j}^{-1}\right)_{j \geq 1}\right\|_{\ell_{q}}^{q} .
$$

Taking the $q$ th root in (3.14) and inserting into (3.13) gives (3.6).

Remark 3.2. Note that the lower set $\Lambda$ guaranteed in the above theorem can be described a priori by choosing the indices corresponding to the $n$ largest of the numbers $\rho^{-\nu}$ with ties handled properly; see also $[4,7]$.

We next want to derive a local version of the last theorem, namely we want to derive an estimate for how well the Taylor series centered at a general point $\bar{y} \in Y$ approximates $u$ near $\bar{y}$. Suppose that $Q_{\lambda}(\bar{y}) \subset Y$ is a hyperrectangle centered at some $\bar{y} \in Y$ with sidelength $2 \lambda_{j}$ in direction $j$, i.e.,

$$
Q_{\lambda}(\bar{y}):=\left\{y \in \mathbb{R}^{\mathbb{N}}: \quad\left|y_{j}-\bar{y}_{j}\right| \leq \lambda_{j}, \quad j \geq 1\right\} .
$$

We refer to the sequence $\lambda:=\left(\lambda_{j}\right)_{j \geq 1}$ as the sidelength vector for this set.

A first local error estimate for the Taylor series at $\bar{y}$ is given in the following corollary. In preparation for the proof of that corollary, let us note that for $y \in Q_{\lambda}(\bar{y})$, we have

$$
a(y)=a(\bar{y})+\sum_{j=1}^{\infty} \frac{\left(y_{j}-\bar{y}_{j}\right)}{\lambda_{j}}\left(\lambda_{j} \psi_{j}\right)=a(\bar{y})+\sum_{j=1}^{\infty} \tilde{y}_{j} \tilde{\psi}_{j}=: \tilde{a}(\tilde{y}),
$$

where $\tilde{y}_{j}:=\frac{y_{j}-\bar{y}_{j}}{\lambda_{j}} \in[-1,1]$ and $\tilde{\psi}_{j}:=\lambda_{j} \psi_{j}$. Therefore,

$$
u(y)=\tilde{u}(\tilde{y}), \quad y \in Q_{\lambda}(\bar{y})
$$

with $\tilde{u}(\tilde{y})$ the solution to

$$
-\operatorname{div}(\tilde{a}(\tilde{y}) \nabla \tilde{u}(\tilde{y}))=f, \quad \tilde{y} \in Y,
$$

in $D$ with Dirichlet homogeneous boundary conditions.

We can now apply Theorem 3.1 to this new problem (3.17) as long as the assumptions of that theorem hold for this new problem.

Corollary 3.3. Suppose the assumptions of Theorem 3.1 hold for $\kappa$ and $\delta$ as in (3.4) and (3.5). Consider any hyperrectangle $Q:=Q_{\lambda}(\bar{y}) \subset Y$ as in (3.15) with center $\bar{y} \in Y$ and sidelength vector $\lambda$. If there is a sequence $\left(\tilde{\rho}_{j}\right)_{j \geq 1}$ (depending on $Q$ ) for which

(i) $\tilde{\rho}_{j} \geq \kappa$ for $j \geq 1$; 
(ii) $\left\|\left(\tilde{\rho}_{j}^{-1}\right)_{j \geq 1}\right\|_{\ell_{q}} \leq\left\|\left(\rho_{j}^{-1}\right)_{j \geq 1}\right\|_{\ell_{q}}$;

(iii) $\left\|\frac{\sum_{j \geq 1} \tilde{\rho}_{j}\left|\tilde{\psi}_{j}\right|}{a(\bar{y})}\right\|_{L^{\infty}(D)} \leq \delta$,

then for each $m \geq 1$, there is a polynomial $P$ (depending on $Q$ ) with $m$ terms (whose indices are given by a lower set) such that

$$
\sup _{y \in Q}\|u(y)-P(y)\|_{V} \leq C(\delta, \rho, q)\left\|\left(\tilde{\rho}_{j}^{-1}\right)_{j \geq 1}\right\|_{\ell_{q}} m^{-r}, \quad r=1 / q-1 / 2,
$$

where $C(\delta, \rho, q)$ is the constant from Theorem 3.1.

Proof. This follows from Theorem 3.1 applied to the new problem (3.17). We obtain the same constant because of the assumptions (i)-(iii) placed on the sequence $\left(\tilde{\rho}_{j}\right)_{j \geq 1}$.

\subsection{A general upper bound on the library size}

We now turn to the central issue: given $m$, and a desired accuracy $\varepsilon$, how can we partition the parameter domain $Y$ into a finite number of cells such that $u$ can be approximated to this accuracy by a piecewise polynomial on this partition, where each polynomial has $m+1$ terms? Deriving such a partition and bounding its size requires some preparatory work. Let $C:=C(\delta, \rho, q)$ be the constant of Theorem 3.1. We assume without loss of generality that

$$
C\left\|\left(\rho_{j}^{-1}\right)_{j \geq 1}\right\|_{\ell_{q}}(m+1)^{-r}>\varepsilon
$$

since otherwise the parameter domain $Y$ does not need to be partitioned. Namely, from Theorem 3.1, there is a polynomial with $m+1$ terms which approximates $u$ on $Y$ to accuracy $\varepsilon$. Since $\left(\rho_{j}^{-1}\right)_{j \geq 1} \in \ell_{q}(\mathbb{N})$, we define $J:=J(\varepsilon, m) \geq 1$ to be the smallest integer such that

$$
\sum_{j \geq J+1} \rho_{j}^{-q} \leq \frac{1}{2} C^{-q}(m+1)^{q r} \varepsilon^{q} .
$$

We will see that the directions $J+1, J+2, J+3, \ldots$, contribute at most $\varepsilon / 2$ to the total error, and we will not need to subdivide in these directions. For the first $J$ directions, we use a strategy that distributes the remaining error equally. To that purpose, we define the quantity

$$
\sigma^{q}:=\frac{1}{2 J} C^{-q}(m+1)^{q r} \varepsilon^{q}
$$

With this notation, we can rewrite (3.19) and (3.20), respectively, as

$$
\left\|\left(\rho_{j}^{-1}\right)_{j \geq 1}\right\|_{\ell_{q}}^{q}>2 J \sigma^{q}, \text { and } \sum_{j \geq J+1} \rho_{j}^{-q} \leq J \sigma^{q} .
$$

We begin with the following lemma.

Lemma 3.4. Suppose $Q \subset Y$ is a hyperrectangle with center $z=\left(z_{1}, \ldots, z_{J}, 0,0, \ldots\right)$ and sidelength vector $\lambda=\left(\lambda_{1}, \ldots, \lambda_{J}, 1,1, \ldots\right)$. If

$$
\lambda_{j} \leq \sigma\left(\rho_{j}-\left|z_{j}\right|\right) \quad j=1, \ldots, J,
$$

then there exists a $V$ valued polynomial $P_{Q}$ with $m+1$ terms such that

$$
\left\|u(y)-P_{Q}(y)\right\|_{V} \leq \varepsilon, \quad y \in Q .
$$


Proof. We define

$$
\tilde{\rho}_{j}:= \begin{cases}\sigma^{-1}, & \text { if } 1 \leq j \leq J, \\ \rho_{j}, & \text { otherwise }\end{cases}
$$

and verify that $\left(\tilde{\rho}_{j}\right)_{j \geq 1}$ satisfies the assumptions (i)-(iii) of Corollary 3.3 for $Q$, beginning with (i). It follows from the definition (3.4) of $\kappa$ and from (3.22) that

$$
\sigma^{q}<\frac{1}{2 J}\left\|\left(\rho_{j}^{-1}\right)_{j \geq 1}\right\|_{\ell_{q}}^{q}=\frac{1}{2 J}\left(\sum_{j=1}^{J} \rho_{j}^{-q}+\sum_{j \geq J+1} \rho_{j}^{-q}\right) \leq \frac{1}{2} \kappa^{-q}+\frac{1}{2} \sigma^{q},
$$

and so $\sigma^{-1}>\kappa$. Since we already assume that $\rho_{j} \geq \kappa$ for all $j$, this verifies condition (i).

To prove (ii), we set $\eta:=C^{-1} \varepsilon(m+1)^{r}$ and use the choice of $J$ in (3.20) to write

$$
\left\|\left(\tilde{\rho}_{j}^{-1}\right)_{j \geq 1}\right\|_{\ell_{q}}^{q}=J \sigma^{q}+\sum_{j \geq J+1} \rho_{j}^{-q} \leq J \sigma^{q}+\frac{1}{2} \eta^{q}=\eta^{q} .
$$

Moreover, if we combine (3.26) with (3.22), we obtain

$$
\left\|\left(\tilde{\rho}_{j}^{-1}\right)_{j \geq 1}\right\|_{\ell_{q}}^{q} \leq \eta^{q}<\left\|\left(\rho_{j}^{-1}\right)_{j \geq 1}\right\|_{\ell_{q}}^{q}
$$

and so (ii) holds.

Finally, to prove (iii), recall that $\tilde{\psi}_{j}=\lambda_{j} \psi_{j}$ and therefore from the inequalities (3.25) and (3.23) we have

$$
\tilde{\rho}_{j}\left|\tilde{\psi}_{j}\right|=\tilde{\rho}_{j} \lambda_{j}\left|\psi_{j}\right| \leq\left(\rho_{j}-\left|z_{j}\right|\right)\left|\psi_{j}\right| .
$$

This gives

$$
\left\|\frac{\sum_{j \geq 1} \tilde{\rho}_{j}\left|\tilde{\psi}_{j}\right|}{a(z)}\right\|_{L^{\infty}(D)} \leq\left\|\frac{\sum_{j \geq 1} \rho_{j}\left|\psi_{j}\right|-\sum_{j \geq 1}\left|z_{j}\right|\left|\psi_{j}\right|}{\bar{a}-\sum_{j \geq 1}\left|z_{j}\right|\left|\psi_{j}\right|}\right\|_{L^{\infty}(D)} \leq \delta .
$$

In view of the definition of $\delta$ in (3.5), the last inequality follows from

$$
0 \leq \sum_{j \geq 1}\left|z_{j}\right|\left|\psi_{j}(x)\right|<\sum_{j \geq 1} \rho_{j}\left|\psi_{j}(x)\right| \leq \bar{a}(x), \quad x \in D,
$$

and the inequality $\left|\frac{\alpha-\beta}{\gamma-\beta}\right| \leq\left|\frac{\alpha}{\gamma}\right|$, which is valid for any $0 \leq \beta<\alpha \leq \gamma$. Thus, (iii) has been established.

We can now use Corollary 3.3 to guarantee the existence of the polynomial $P_{Q}$ to complete the proof.

We are now in position to state the main theorem of this section.

Theorem 3.5. Let $0<q<2$, and let $\left(\rho_{j}^{-1}\right)_{j \geq 1} \in \ell_{q}(\mathbb{N})$ be a nondecreasing sequence which satisfies (3.4) and (3.5). Let $\varepsilon>0, m \geq 0$ and assume that (3.19) holds. Then, there exists a tensor product partition of $Y$ into a collection $\mathcal{R}$ of $N$ hyperrectangles such that on each $Q \in \mathcal{R}$ there is a $V$ valued polynomial $P_{Q}$ with $m+1$ terms such that

$$
\left\|u(y)-P_{Q}(y)\right\|_{V} \leq \varepsilon, \quad y \in Q .
$$

Furthermore, if $J:=J(\varepsilon, m)$ is as in (3.20), then the partition is obtained by only subdividing in the first $J$ directions, and the number of cells $N$ in this partition satisfies

$$
N \leq \prod_{j=1}^{J}\left(\sigma^{-1}\left|\ln \left(1-\rho_{j}^{-1}\right)\right|+C(\sigma)\right) \quad \text { for some } \quad C(\sigma) \in(1,2) .
$$


Proof. To define our tensor product grid, for each coordinate direction $y_{j}, j=1, \ldots, J$, we first define how we subdivide the interval $[-1,1]$ into $\left(2 k_{j}+1\right)$ subintervals

$$
I_{j}^{i}, \quad-k_{j} \leq i \leq k_{j} .
$$

Recall that we do not subdivide any of the coordinate axis when $j>J$, i.e., $k_{j}=0$ and $I_{j}^{0}=[-1,1]$ when $j>J$. Also, our partition is symmetric and so $I_{j}^{-i}=-I_{j}^{i}, i=1, \ldots, k_{j}$.

We fix $j \in\{1, \ldots, J\}$ and describe our partition of $[-1,1]$ into intervals corresponding to the $j$-th coordinate. Our first interval $I_{j}^{0}$ is centered at $z_{j}^{0}=0$ and has sidelength $\lambda_{j}^{0}:=\sigma \rho_{j}$ provided this number is less than one. Otherwise, when $\sigma \rho_{j} \geq 1$, we define $\lambda_{j}^{0}:=1$, and so $k_{j}=0$ and our partition consists only of the one interval $I_{j}^{0}=[-1,1]$. Note that since $\left(\rho_{j}\right)_{j \geq 1}$ is nondecreasing, when this occurs it also happens for all larger values of $j$.

Our partition is symmetric with respect to the origin and so we only describe the intervals to the right of the origin. The next interval $I_{j}^{1}$ has left endpoint the same as the right endpoint of $I_{j}^{0}$, has center $z_{j}^{1}$ and sidelength $\lambda_{j}^{1}$, where these numbers are defined by the relationship

$$
\lambda_{j}^{1}=\sigma\left(\rho_{j}-z_{j}^{1}\right) .
$$

The only exception to this definition is when the right endpoint of this interval is larger than 1 . Then we recenter the interval so its left endpoint is as before and its right endpoint is 1 . In this case, we would stop the process and $k_{j}$ would be 1 .

We continue in this same way moving to the right. In general, the interval $I_{j}^{i}$ will have its left endpoint equal to the right endpoint of $I_{j}^{i-1}$, with center $z_{j}^{i}$ and sidelength $\lambda_{j}^{i}$ which satisfy

$$
\lambda_{j}^{i}=\sigma\left(\rho_{j}-z_{j}^{i}\right)
$$

As before, we rescale in the case that such a choice would give a right endpoint larger than 1 and terminate the partitioning process. It follows that the interval $I_{j}^{i}$ always satisfies

$$
\lambda_{j}^{i} \leq \sigma\left(\rho_{j}-z_{j}^{i}\right), \quad i=0,1, \ldots, k_{j},
$$

with equality except for possibly the last interval $I_{j}^{k_{j}}$. We give below a bound for $k_{j}$ that shows this process is finite.

This partitioning gives a tensor product set $\mathcal{R}$ of hyperrectangles $Q$. In view of the property (3.32), each of the hyperrectangles satisfies the conditions of Lemma 3.4 and therefore the existence of the polynomials $P_{Q}$, $Q \in \mathcal{R}$ satisfying the approximation estimate is guaranteed.

We next derive a bound for the number of elements $\mathcal{R}$ by bounding $n_{j}=2 k_{j}+1,1 \leq j \leq J$, when $k_{j} \neq 0$. To do that, we monitor the points

$$
R^{i}=z_{j}^{i}+\lambda_{j}^{i}, \quad i=0,1, \ldots, k_{j} .
$$

Each $R^{i}$ is the right endpoint of $I_{j}^{i}$ as long as $0 \leq i<k_{j}$, and we know that $R^{k_{j}} \geq 1$. Relation (3.31) implies that $\lambda_{j}^{i}$ is chosen according to

$$
\frac{\lambda_{j}^{i}}{\rho_{j}-R^{i}+\lambda_{j}^{i}}=\sigma .
$$

This gives that $(1-\sigma) \lambda_{j}^{i}=\sigma\left(\rho_{j}-R^{i}\right)$, and since $R^{i}=R^{i-1}+2 \lambda_{j}^{i}$, we have

$$
(1-\sigma)\left(R^{i}-R^{i-1}\right)=2 \sigma\left(\rho_{j}-R^{i}\right) .
$$

We therefore obtain the recursive formula

$$
R^{i}=\frac{1-\sigma}{1+\sigma} R^{i-1}+\frac{2 \sigma}{1+\sigma} \rho_{j}=: \alpha R^{i-1}+b, \quad i=1,2, \ldots,
$$


where $R^{0}=\rho_{j} \sigma, \alpha:=\frac{1-\sigma}{1+\sigma}, b:=\frac{2 \sigma}{1+\sigma} \rho_{j}$. Therefore, we find

$$
\begin{aligned}
R^{i} & =\alpha^{i} R^{0}+\left(1+\alpha+\ldots+\alpha^{i-1}\right) b=\alpha^{i} R^{0}+\frac{1-\alpha^{i}}{1-\alpha} b \\
& =\alpha^{i} \rho_{j} \sigma+\left(1-\alpha^{i}\right) \rho_{j}=\rho_{j}\left(1-\alpha^{i}(1-\sigma)\right) .
\end{aligned}
$$

The iteration will stop at the smallest integer $k=k_{j}$ with the property $R^{k} \geq 1$. Since $\sigma^{-1} \geq \kappa>1$, we have $\sigma<1$ and the iteration will stop at the smallest integer $k$ such that

$$
\alpha^{k} \leq \frac{1-\rho_{j}^{-1}}{1-\sigma} .
$$

Note that $\frac{1-\rho_{j}^{-1}}{1-\sigma}<1$ because $\sigma \rho_{j}<1$ (otherwise $k_{j}=0$ and $I_{j}^{0}=[-1,1]$ ). We are looking for the smallest integer $k$ for which

$$
k \geq \frac{\ln \left(1-\rho_{j}^{-1}\right)-\ln (1-\sigma)}{\ln \alpha},
$$

which is given by

$$
k_{j}=\left\lceil\frac{\ln \left(1-\rho_{j}^{-1}\right)-\ln (1-\sigma)}{\ln \alpha}\right\rceil<\frac{\ln \left(1-\rho_{j}^{-1}\right)-\ln (1-\sigma)}{\ln \alpha}+1, \quad j=1, \ldots, J .
$$

Therefore, we have the bound

$$
n_{j}:=2 k_{j}+1 \leq 2 \frac{\ln \left(1-\rho_{j}^{-1}\right)-\ln (1-\sigma)}{\ln \left(\frac{1-\sigma}{1+\sigma}\right)}+3=2 \frac{\ln \left(1-\rho_{j}^{-1}\right)}{\ln \left(\frac{1-\sigma}{1+\sigma}\right)}+C(\sigma),
$$

where

$$
C(\sigma):=-2 \frac{\ln (1-\sigma)}{\ln \left(\frac{1-\sigma}{1+\sigma}\right)}+3=\frac{\ln \left(\frac{(1-\sigma)}{(1+\sigma)^{3}}\right)}{\ln \left(\frac{1-\sigma}{1+\sigma}\right)} \in(1,2) .
$$

Since $\ln (1+x) \geq \frac{2 x}{2+x}$ for $x \geq 0$, we obtain

$$
\ln \left(\frac{1+\sigma}{1-\sigma}\right)=\ln \left(1+\frac{2 \sigma}{1-\sigma}\right) \geq 2 \sigma,
$$

and thus $n_{j} \leq \sigma^{-1}\left|\ln \left(1-\rho_{j}^{-1}\right)\right|+C(\sigma)$, which brings us to the final calculation

$$
N=\prod_{j=1}^{J} n_{j} \leq \prod_{j=1}^{J}\left(\sigma^{-1}\left|\ln \left(1-\rho_{j}^{-1}\right)\right|+C(\sigma)\right),
$$

which completes the proof.

Remark 3.6. It follows from the proof of Theorem 3.5 that a more precise estimate for the number of cells is

$$
N \leq \prod_{j=1}^{J_{0}}\left(\sigma^{-1}\left|\ln \left(1-\rho_{j}^{-1}\right)\right|+C(\sigma)\right)
$$

where $1 \leq J_{0} \leq J$ is the largest integer such that $\sigma \rho_{J_{0}}<1$. This comes from the fact that $k_{j}=0$ for $J_{0}<j \leq J$, i.e., we do not subdivide in the directions $J_{0}+1 \ldots, J$. 
Let us reformulate the above result in terms of library widths. As we have remarked earlier (see (3.3)), a polynomial approximation with $m+1$ terms is naturally associated with an affine space of dimension at most $m$. We then obtain a library $\mathcal{L}=\cup_{i=1}^{N} L_{i}$ of affine spaces $L_{i}=L_{i}\left(P_{i}, Q_{i}\right)$,

$$
L_{i}=c_{0}^{i}+\operatorname{span}\left\{c_{\nu}^{i} \in V: \nu \in \Lambda_{i}, \#\left(\Lambda_{i}\right) \leq m\right\}, \quad i=1, \ldots, N,
$$

each associated with the polynomial $P_{i}$ over a hyperrectangle $Q_{i} \subset Y$,

$$
P_{i}(y)=c_{0}^{i}+\sum_{\nu \in \Lambda_{i}} c_{\nu}^{i} y^{\nu}, \quad y \in Q_{i}
$$

and cardinality

$$
N \leq \prod_{j=1}^{J}\left(\sigma^{-1}\left|\ln \left(1-\rho_{j}^{-1}\right)\right|+C(\sigma)\right), \quad C(\sigma) \in(1,2) .
$$

Moreover, $\operatorname{since}_{\sup _{y \in Q_{i}}}\left\|u(y)-P_{i}(y)\right\|_{V} \leq \varepsilon$ for $i=1, \ldots, N$, we have

$$
E_{\mathcal{L}}(\mathcal{M})=\max _{y \in Y} \min _{L \in \mathcal{L}} \operatorname{dist}(u(y), L)_{V} \leq \varepsilon
$$

and therefore

$$
d_{m, k}(\mathcal{M}) \leq \varepsilon, \quad \text { whenever } \quad k \geq \prod_{j=1}^{J}\left(\sigma^{-1}\left|\ln \left(1-\rho_{j}^{-1}\right)\right|+C(\sigma)\right) .
$$

\subsection{An upper bound on the library size for some specific examples}

To see how how the bounds for $N$ grow with decreasing $\varepsilon$ in Theorem 3.5, we consider the following standard example:

$$
\rho_{j}=M j^{s}, \quad j \geq 1,
$$

where $s>1 / 2$ is fixed. From our assumption that $\kappa=\rho_{1}>1$, it follows that $M>1$. We note at the outset that a similar analysis can be done for other growth assumptions on the sequence $\left(\rho_{j}\right)_{j \geq 1}$, e.g., $\rho_{j}=1+M j^{s}$ with $M>0$.

Before beginning our analysis, we wish to orient the reader to what type of results we can expect by reflecting on the corresponding results for polynomial approximation. In that case, we know that for each $r<s-1 / 2$ we can find $V$ valued polynomials $P_{n}$ with $n$ terms that satisfy

$$
\max _{y \in Y}\left\|u(y)-P_{n}(y)\right\|_{V} \leq C_{r} n^{-r}, \quad n=1,2, \ldots
$$

This follows from Theorem 3.1 by choosing a value of $q \in(1 / s, 2)$ with $r=1 / q-1 / 2$. However, we cannot take $r=s-1 / 2$ since the constants $C_{r}$ tend to infinity as $q \rightarrow 1 / s$. If we are given a target accuracy $\varepsilon$ then we would find the minimal number of terms $n$ to reach this accuracy by optimizing over the choice of $q$. This type of analysis is subtle and done in [4].

We shall obtain similar results for piecewise polynomial approximation where now the main new ingredient is to bound the number of cells that are needed. We fix the desired target accuracy $\varepsilon>0$ and the value $m$ and use the a priori bound of Theorem 3.5 to see how many hyperrectangles $N$ are needed to guarantee the accuracy $\varepsilon$ using piecewise polynomials with $m+1$ terms to approximate $u$ on each rectangle. We can apply Theorem 3.5 for any $q$ that satisfies $1 / s<q<2$, and for the moment we fix any such $q$. Throughout the derivation, we let $C$ denote a constant that depends only on $q$ and may change from line to line. Note that $C_{0}:=C(\delta, \rho, q)$ depends only on $q$ since $\rho$ and $\delta$ are fixed.

Since we have

$$
\sum_{j \geq J+1} \rho_{j}^{-q}=M^{-q} \sum_{j \geq J+1} j^{-s q} \leq C J^{1-s q},
$$


the condition (3.20) is satisfied if

$$
J=C\left(\varepsilon(m+1)^{r}\right)^{\frac{q}{1-s q}}=C \lambda^{\frac{q}{1-s q}}
$$

where

$$
\lambda:=\varepsilon(m+1)^{r}, \quad r=r(q):=\frac{1}{q}-\frac{1}{2} .
$$

Defining $J$ by (3.38) gives that the value of $\sigma$ in the theorem is

$$
\sigma=2^{-1 / q} C_{0}^{-1} J^{-1 / q} \lambda=C J^{-s} .
$$

Theorem 3.5 says that we obtain a partition into $N$ hyperrectangular cells such that there is a polynomial with $m+1$ terms on each cell which achieves the desired accuracy $\varepsilon$. It also gives that the number $N=N(q)$ of these cells can be bounded by

$$
N \leq \prod_{j=1}^{J}\left(\sigma^{-1}\left|\ln \left(1-\rho_{j}^{-1}\right)\right|+C(\sigma)\right)<\prod_{j=1}^{J}\left(\sigma^{-1}\left|\ln \left(1-\rho_{j}^{-1}\right)\right|+2\right) .
$$

Since each $\rho_{j} \geq M>1$, and $|\ln (1-x)| \leq \frac{x}{1-x}$, for $0<x<1$, we have

$$
\left|\ln \left(1-\rho_{j}^{-1}\right)\right| \leq\left(M j^{s}-1\right)^{-1} \leq(M-1)^{-1} j^{-s}, \quad j=1,2, \ldots .
$$

Placing this into (3.40) gives

$$
N \leq \prod_{j=1}^{J}\left((M-1)^{-1} \sigma^{-1} j^{-s}+2\right)=\prod_{j=1}^{J}\left(C J^{s} j^{-s}+2\right) \leq C^{J} J^{s J}[J !]^{-s} \leq e^{(C+s) J}=e^{C \lambda^{\frac{q}{1-s q}}},
$$

where the last inequality uses Stirling's formula.

We examine what this bound guarantees for different values of $m$ :

Case $\boldsymbol{m}=\mathbf{0}$. In this case, since $\lambda=\varepsilon$, we are providing the solution manifold $\mathcal{M}$ with an $\varepsilon$ approximation net of $N$ elements, with

$$
N \leq \exp \left\{C \varepsilon^{-\frac{1}{s-1 / q}}\right\},
$$

for any $q \in(1 / s, 2)$. The best choice of $q$ in this case is to choose $q$ as close to 2 as possible thereby getting $N \leq e^{C \varepsilon^{-1 / \alpha}}$ for any $0<\alpha<s-1 / 2$. Notice that this is in complete agreement with what we know about the entropy of the solution manifold $\mathcal{M}$. Indeed, from Theorem 3.1, we know the Kolmogorov $n$ width of $\mathcal{M}$ satisfies

$$
d_{n}(\mathcal{M}) \leq C_{r} M n^{-r}, \quad 0<r<s-1 / 2,
$$

where the constants $C_{r}$ tend to infinity as $r$ gets closer to $s-1 / 2$. From Carl's inequality we obtain that the $\varepsilon$ covering number of $\mathcal{M}$ is bounded by $e^{C \varepsilon^{-1 / r}}$ provided that $r<s-1 / 2$, which is exactly what the above bound on $N$ gives.

Case of general $\boldsymbol{m}$. In this case, the partitioning gives a library of $N$ affine spaces of dimension $m$ that approximate $\mathcal{M}$ to accuracy $\varepsilon$. In order to compare our results on piecewise polynomial approximation with those for polynomial approximation, we suppose a value of $q \in(1 / s, 2)$ has been chosen which gives the polynomial approximation accuracy $C_{r} n^{-r}$, with $r=r(q)=1 / q-1 / 2$. As described as in Theorem 3.5, we obtain the same accuracy $\varepsilon:=C_{r} n^{-r}$ using a piecewise polynomial defined over

$$
N \leq \exp \left\{C\left(\frac{n}{m+1}\right)^{\frac{r}{s-1 / q}}\right\}=\exp \left\{C\left(\frac{n}{m+1}\right)^{\alpha}\right\}, \quad \alpha:=\frac{1 / q-1 / 2}{s-1 / q}
$$


cells, with $m+1$ polynomial terms on each cell. Notice that rather than the bound $e^{C(n-m)}$ derived in Section 2 for general libraries, we now have the bound $e^{C(n / m)^{\alpha}}$, which gets more favorable as $m$ gets large. We can always get $\alpha=1$ by taking $q=\frac{4}{2 s+1}$, which belongs to the prescribed range $(1 / s, 2)$, since $s>1 / 2$ by assumption. Moreover, $\alpha$ tends to infinity as $q \rightarrow 1 / s$ and to 0 as $q \rightarrow 2$.

\section{Numerical EXAMPLES}

In this section, we present numerical examples to illustrate the performance of nonlinear reduced models. Our strategy is based on a partitioning of the parameter domain $Y$ and using piecewise $V$ valued polynomials subordinate to the chosen partition. For our numerical tests, we consider the elliptic equations (1.6) on the domain $D=[0,1]^{2}$ with right-hand side $f=1$ and an affine diffusion of the form

$$
a(x, y):=1+\sum_{j=1}^{64} y_{j} c_{j} \chi_{D_{j}}(x), \quad x \in D, \quad y \in Y=[-1,1]^{64},
$$

where $\left(D_{j}\right)_{j=1}^{64}$ is a partition of $D$ into 64 square cells of equal size. The indexing is assigned randomly and has little effect on the numerical results.

We carry out numerical experiments for different sequences $\left(c_{j}\right)_{j=1}^{64}$ that depend on the parameters $a_{\min }$ and $s$, namely

$$
c_{j}=\left(1-a_{\min }\right) j^{-s}, \quad j=1,2, \ldots, 64,
$$

where $s \in\{2,3,4\}$ and $a_{\min } \in\{0.1,0.05,0.01\}$. Each numerical experiment corresponds to an assignment of $a_{\min }$ and $s$. Given this sequence $\left(c_{j}\right)_{j=1}^{64}$, we can take

$$
\rho_{j}:=\frac{1-a_{\min } / 2}{1-a_{\min }} j^{s}, \quad j=1,2, \ldots, 64,
$$

which determines the value of $\delta=1-\frac{a_{\min }}{2}$ in (3.5). Notice that $a_{\min }$ is the true minimum of $a$ on $D \times Y$, and a small value for $a_{\min }$ corresponds to a reduction in the domain of analyticity of $u(y)$ near the face $y_{1}=-1$.

\subsection{Linear reduced models}

We begin this section by considering linear reduced models with the goal of understanding how large the dimension of the linear space has to be in order to guarantee a prescribed error $\varepsilon$. We are also interested to see the effect of different choices for the linear space. In all of our numerical experiments we take the target error to be

$$
\varepsilon:=10^{-4} \text {. }
$$

We consider two choices of linear reduced models: (1) Taylor polynomial spaces, and (2) reduced basis spaces based on greedily selected snapshots. We compare the approximations obtained using a Taylor polynomial with $n$ terms and a reduced basis space of dimension $n$. In particular, we want to see how large $n$ has to be to achieve the target accuracy $\varepsilon$ for these two choices.

In the case of a Taylor polynomial space, the approximation $\bar{u}_{n}$ is given by

$$
\bar{u}_{n}(y):=\bar{t}_{0}+\sum_{\nu \in \Lambda_{n}^{*}} \bar{t}_{\nu} y^{\nu} \in \bar{t}_{0}+V_{n-1}(T), \quad V_{n-1}(T):=\operatorname{span}\left\{\bar{t}_{\nu}: \nu \in \Lambda_{n}^{*}\right\},
$$

where $\bar{t}_{\nu}$ is the approximation of $t_{\nu}$ obtained using a finite element solver of high accuracy (much higher accuracy than the target accuracy $\varepsilon$ ). We consider two methods to generate the lower set $\Lambda_{n}^{*}$ of cardinality $n-1$ which gives the indices $\nu$ in (4.4).

The first method, which we refer to as the a priori method, orders the $\rho^{-\nu}, \nu \in \mathcal{F}$, in decreasing order according to their size. So $\nu^{0}:=0$ is the index giving the largest of these numbers, and $\nu^{1}, \nu^{2}, \ldots$ denote the 

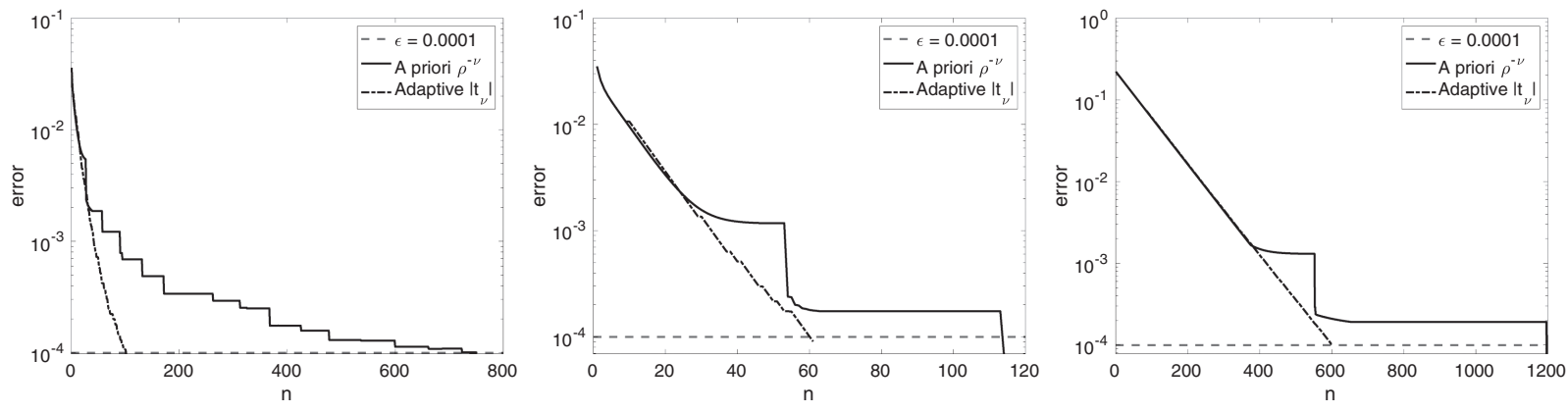

FigURE 1. Error between $\bar{u}$ and the Taylor polynomial approximation $\bar{u}_{n}$ versus the number of terms $n$ for both the a priori method and the adaptive method for constructing $\Lambda_{n}^{*}$. Left: $s=2$, $a_{\min }=0.1 ;$ middle: $s=4, a_{\min }=0.1 ;$ right: $s=4, a_{\min }=0.01$.

indices corresponding to the next largest of the $\rho^{-\nu}$. Ties are handled in such a way that $\Lambda_{n}:=\left\{\nu^{0}, \nu^{1}, \ldots, \nu^{n-1}\right\}$ is a lower set, see [4]. We then take $\Lambda_{n}^{*}:=\Lambda_{n} \backslash\left\{\nu^{0}\right\}$.

In the second method, here referred to as the adaptive method, we use the so-called Algorithm LN (largest neighbor) described in [5] to generate an index set $\tilde{\Lambda}_{n}$. It begins with $\nu^{0}:=0$ and $\tilde{\Lambda}_{0}:=\left\{\nu^{0}\right\}$. Then, for $k=0,1, \ldots, n-1$,

$$
\tilde{\Lambda}_{k+1}:=\tilde{\Lambda}_{k} \cup\left\{\nu^{k}\right\}, \quad \text { where } \quad \nu^{k} \in \operatorname{argmax}_{\nu \in \mathcal{R}_{\tilde{\Lambda}_{k}}}\left\|\bar{t}_{\nu}\right\|_{V} .
$$

Here, $\mathcal{R}_{\tilde{\Lambda}_{k}}$ denotes the reduced margin of the current lower set $\tilde{\Lambda}_{k}$, namely

$$
\mathcal{R}_{\tilde{\Lambda}_{k}}:=\left\{\nu \in \mathcal{F} \backslash \tilde{\Lambda}_{k}: \nu-e_{j} \in \tilde{\Lambda}_{k} \quad \text { for all } j \text { with } \nu_{j}>0\right\} .
$$

We then take $\Lambda_{n}^{*}:=\tilde{\Lambda}_{n} \backslash\left\{\nu^{0}\right\}$.

We compute the error $\epsilon_{n}$ for each of these choices by taking a large number of uniform random choices ${ }^{1}$ of parameters $y \in Y$, as follows. For each choice $y$, we take an accurate finite element approximation $\bar{u}(y)$ of $u(y)$ as truth. Note that because $\Lambda_{n}^{*} \cup\{0\}$ is a lower set, the Taylor coefficients $t_{\nu}, \nu \in \Lambda_{n}^{*} \cup\{0\}$, can be found recursively, see equations (3.1) and (3.2) in [5]. We calculate $\left\|\bar{u}(y)-\bar{u}_{n}(y)\right\|_{V}$ and the error $\epsilon_{n}$ is then computed by maximizing $\left\|\bar{u}(y)-\bar{u}_{n}(y)\right\|_{V}$ over the random choices of $y$.

Figure 1 shows a comparison of the errors obtained using the adaptive and the a priori methods to compute the set $\Lambda_{n}^{*}$ as $n$ grows for different values of $s$ and $a_{\text {min }}$. We see that the adaptive method to generate $\Lambda_{n}^{*}$ outperforms the a a priori method, in that the corresponding approximation error is smaller for the adaptive method. This is caused by the fact that $\left\|\bar{t}_{\nu}\right\|_{V}$ could be much smaller than $\rho^{-\nu}$. On the other hand, the computational cost to find $\Lambda_{n}^{*}$ is greater for the adaptive method. In going further in this section, we always compute the set $\Lambda_{n}^{*}$ for Taylor polynomial indices by using the adaptive method.

We next discuss greedy basis constructions. In this case, the reduced linear space $V_{n}(G)$ is constructed by starting with the function $\varphi_{0}:=u(0)$ and then using a particular random weak greedy algorithm ${ }^{2}$ to generate the reduced basis functions $\varphi_{1}, \ldots, \varphi_{n-1}$. Each $\varphi_{j}$ is a snapshot $\varphi_{j}=u\left(y^{(j)}\right)$ of the solution at a judiciously chosen point $y^{(j)} \in Y$. We denote by $\bar{\varphi}_{j}$ an accurate finite element approximation of $\varphi_{j}, j=0,1, \ldots, n-1$, and we define $V_{n}(G):=\operatorname{span}\left\{\bar{\varphi}_{0}, \bar{\varphi}_{1}, \ldots, \bar{\varphi}_{n-1}\right\}$. The reduced model is now

$$
\bar{u}_{n}(y):=P_{V_{n}}(u(y)),
$$

\footnotetext{
${ }^{1}$ In the experiments the number of random selections of $y$ was $10^{3}$, using the Mersenne Twister pseudo random generator with seed value 515 .

${ }^{2}$ We use a version of the probabilistic weak greedy algorithm given in [8].
} 

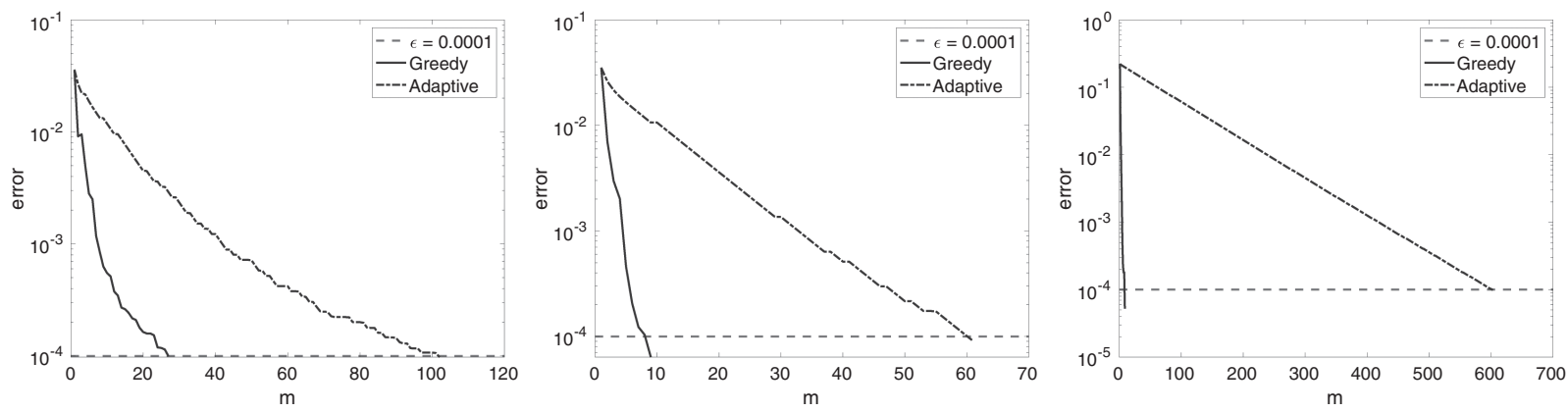

FigURE 2. Error between $\bar{u}$ and $\bar{u}_{n}$ versus $n$ for both the (adaptive) Taylor and greedy reduced models. Left: $s=2, a_{\min }=0.1 ;$ middle: $s=4, a_{\min }=0.1 ;$ right: $s=4, a_{\min }=0.01$.

where $P_{V_{n}}$ is the Galerkin projection onto $V_{n}(\mathrm{G})$. Namely, for a given $y \in Y, \bar{u}_{n}(y) \in V_{n}(G)$ is the solution of

$$
\int_{D} a(\cdot, y) \nabla \bar{u}_{n}(y) \cdot \nabla \bar{v}_{n}=\int_{D} f \bar{v}_{n}, \quad \bar{v}_{n} \in V_{n}(G) .
$$

We compute the error for approximating $u(y)$ using random samples of the parameter $y$ in a similar manner to the Taylor case already discussed.

Figure 2 gives a comparison of the performance of the greedy basis and the (adaptive) Taylor for different values of $s$ and $a_{\min }$. This graph shows that the greedy basis produces a much more accurate reduced model than the Taylor basis given the same allocation $n$ for the dimension of the reduced space.

\subsection{Nonlinear models based on piecewise polynomials}

The next set of experiments numerically implements a strategy for generating a nonlinear reduced model based on piecewise polynomials similar to that described in Section 3. We consider the same diffusion coefficients as above and the same values of $s$ and $a_{\min }$.

We again fix a target accuracy $\varepsilon=10^{-4}$, and a target value of $m$ for the dimension of the polynomial space on each cell of the partition. We will see that it is not always possible to achieve a partition of reasonable size if $m$ is chosen to be too small. This is heuristically clear from the entropy considerations provided in Sections 2 and 3.3.

Our strategy for generating the partitioning of $Y$ into cells is motivated by the theoretical results of Section 3 . However, we make some modifications of this strategy which we now explain. Since in our numerical examples $u$ has singularity near $y=-1$ because $c_{j}>0$ for all $j=1, \ldots, 64$, we now grade the partition to be finer near -1 when we refine a coordinate direction. This is in contrast to the theoretical description, which partitions in a symmetric way for each coordinate $y_{j}$.

On the other hand, we have found that prescribing $\varepsilon$ and $m$ and then implementing the theoretical partitioning strategy actually produces a partition with much better accuracy than $\varepsilon$, and thus we have used too many cells. So instead of viewing the target error and $m$ as the parameters to determine the partition, we introduce a single parameter $\eta$ to generate a partition. We then select $\eta$ to give the required accuracy $\varepsilon=10^{-4}$ and a good control on $m$ and the number of cells $N$. To be precise, we take $q=1$ and given $\eta>0$, we generate a partition as follows.

\section{Construction of the partition for a given $\eta$ and a non-decreasing sequence $\left(\rho_{j}\right)_{j \geq 1}$}

Choose $J \geq 0$ as the smallest integer such that $\sum_{j=J+1}^{64} \rho_{j}^{-1} \leq \frac{1}{2} \eta$ and set $\sigma=\frac{\eta}{2 J}, j=1$;

While $\sigma \rho_{j}<1$ do

$$
y_{j}^{0}=\frac{1-\sigma \rho_{j}}{1+\sigma}, \lambda_{j}^{0}=\sigma\left(\rho_{j}+y_{j}^{0}\right), i=0 ;
$$

While $y_{j}^{i}-\lambda_{j}^{i}>-1$ 
TABLE 1. Number of terms $m$ needed to meet the target accuracy $\varepsilon=10^{-4}$ on each cell using the piecewise (adaptive) Taylor polynomial approximations.

\begin{tabular}{llllllll}
\hline \hline \multicolumn{3}{c}{$a_{\text {min }}=0.1$} & \multicolumn{5}{c}{$a_{\min }=0.01$} \\
\hline \# of cells & $s=2$ & $s=3$ & $s=4$ & \# of cells & $s=2$ & $s=3$ & $s=4$ \\
\hline$N=1$ & 102 & 68 & 61 & $N=1$ & 666 & 614 & 603 \\
$N=3$ & 29 & 13 & 9 & $N=3$ & 48 & 30 & 27 \\
$N=8$ & 22 & 8 & 5 & $N=10$ & 24 & 11 & 8 \\
\hline
\end{tabular}

Increment $i$; End do$$
\text { Compute } \lambda_{j}^{i}=\frac{\sigma}{1+\sigma}\left(\rho_{j}+y_{j}^{i-1}-\lambda_{j}^{i-1}\right)=\frac{1-\sigma}{1+\sigma} \lambda_{j}^{i-1} \text { and } y_{j}^{i}=y_{j}^{i-1}-\lambda_{j}^{i-1}-\lambda_{j}^{i} \text {; }
$$

If $y_{j}^{i}-\lambda_{j}^{i}<-1$ set $\lambda_{j}^{i}=\frac{1}{2}\left(y_{j}^{i-1}-\lambda_{j}^{i-1}+1\right)$ and $y_{j}^{i}=\frac{1}{2}\left(y_{j}^{i-1}-\lambda_{j}^{i-1}-1\right)$; Increment $j$;

End do

Set $y_{l}^{0}=0, \lambda_{l}^{0}=1$ for $l=j, \ldots, J$.

The algorithm generates a tensor product partition with cells $Q_{\lambda}(\bar{y})$ of the form (3.15). For each cell $Q_{\lambda}(\bar{y})$ from this partition we define a sequence $\left(\tilde{\rho}_{j}\right)_{j \geq 1}$, where

$$
\tilde{\rho}_{j}:= \begin{cases}\frac{\rho_{j}+\bar{y}_{j}}{\lambda_{j}}, & \text { when } \sigma \rho_{j}<1, \\ \rho_{j}, & \text { otherwise. }\end{cases}
$$

It is easy to check that conditions similar to those in Corollary 3.3 are satisfied. Namely, $\tilde{\rho}_{j} \geq \kappa, j=1, \ldots, 64$, and $\left\|\left(\tilde{\rho}_{j}^{-1}\right)_{j=1}^{64}\right\|_{\ell_{q}} \leq\left\|\left(\rho_{j}^{-1}\right)_{j=1}^{64}\right\|_{\ell_{q}}$. Moreover, we have

$$
\tilde{\delta}:=\max _{j=1, \ldots, 64}\left|\frac{\rho_{j} c_{j}+\bar{y}_{j} c_{j}}{1+\bar{y}_{j} c_{j}}\right|<1,
$$

since $\rho_{j} c_{j}=1-a_{\min } / 2<1$, but not necessarily that $\tilde{\delta} \leq \delta$. However, we can still get the error bound (3.18) of Corollary 3.3, but with constant $C(\delta, \rho, q)$ replaced by the potentially larger constant $C(\tilde{\delta}, \rho, q)$. A uniform error bound can be obtained by taking the constant associated to the largest $\tilde{\delta}$ over all cells in the partition.

Table 1 shows the number of terms $m$ needed in the Taylor expansion on each of the $N$ cells from our partition to meet our error criteria. We see that partitioning can significantly reduce the number of polynomial terms needed to meet the target accuracy. For example, in the case $N=1$ (no partitioning), we need to take $m=603$, whereas with only ten cells the necessary $m$ is reduced to 8 . Note however, that reducing $m$ even further may cause a considerable growth in the number of cells $N$. Finally, we mention that $J=1$ in all the examples above.

Remark 4.1. In the above numerical examples, we have not considered the case of using nonlinear models based on piecewise greedy bases. Our current strategy uses the a priori local polynomial error to partition, and we do not yet have a corresponding way to generate a partition of $Y$ when greedy bases are used on each cell. One appropriate strategy would be to do the partitioning in tandem with the local greedy constructions. Strategies for doing this are currently under investigation.

\subsection{State estimation using linear and nonlinear reduced models}

As remarked in the introduction, we anticipate that one of the major advantages of using library approximation occurs in the problem of state estimation from data observations. In this section, we recall the state estimation problem and execute several numerical experiments indicating the performance of piecewise polynomial approximations for this problem. 
In state estimation, we are given measurements of an unknown state $u\left(y^{*}\right)$ where $u$ is the solution to (1.6) with the model $a$ for the diffusion known to us. We assume that the data is of the form

$$
w_{j}=l_{j}\left(u\left(y^{*}\right)\right), \quad j=1, \ldots, L,
$$

where the $l_{j}$ are linear functionals defined on $V$. Each linear functional $l_{j}$ has a Riesz representation

$$
l_{j}(v)=\left\langle v, \omega_{j}\right\rangle_{V}, \quad j=1, \ldots, L .
$$

The functions $\omega_{j}, j=1, \ldots, L$, span a subspace $W$ of $V$. Without loss of generality, we can assume that the dimension of $W$ is $L$ since otherwise there is redundancy in the measurements.

We want to use these data observations together with the known model $a$ for diffusion in order to construct an approximation $\hat{u}$ to the state $u\left(y^{*}\right)$. Note that $y^{*}$ and $u\left(y^{*}\right)$ are not necessarily uniquely determined by the measurements. One way of proceeding, as was proposed in [13], is to employ a reduced model based on a linear space $V_{n}$ to approximate $\mathcal{M}$. The algorithm in [13] constructs an approximation $\hat{u}_{n}$ to $u\left(y^{*}\right)$ by solving a least squares fit to the data from $V_{n}$. This algorithm was shown to be optimal in a certain sense (see $\left.[2,10]\right)$ once $V_{n}$ is chosen. The performance of this algorithm is upper bounded by

$$
\left\|u\left(y^{*}\right)-\hat{u}_{n}\right\|_{V} \leq \mu_{n} \varepsilon_{n} .
$$

Here $\varepsilon_{n}:=\operatorname{dist}\left(\mathcal{M}, V_{n}\right)_{V}$, and $\mu_{n}=\mu\left(W, V_{n}\right) \geq 1$ is a certain inf-sup constant which can be interpreted as the reciprocal of the angle between $V_{n}$ and the space $W$ [3]. More preceisely,

$$
\mu_{n}=\mu\left(W, V_{n}\right):=\left(\inf _{v \in V_{n}} \sup _{w \in W} \frac{\langle v, w\rangle_{V}}{\|v\|_{V}\|w\|_{V}}\right)^{-1} .
$$

This motivates choosing a nested sequence of spaces $V_{1} \subset V_{2} \subset \cdots$ with $\operatorname{dim}\left(V_{j}\right)=j$, and selecting a space from this sequence which minimizes the right side of (4.7). Note that while $\varepsilon_{n}$ decreases when increasing $n$, the constant $\mu_{n}$ increases and is in fact infinite if $n>L$.

For our numerical experiments in state estimation we use the same models for the diffusion $a$ as described in (4.1)-(4.3). For the measurements, we take linear functionals which emulate point evaluation. Specifically, each $l_{j}$ is of the form

$$
l_{j}(u):=\int_{D} u(x) K\left(x-x_{j}\right) \mathrm{d} x, \quad K(x):=\exp \left(-\lambda|x|^{2}\right),
$$

where $|x|$ is the Euclidean norm of $x$ and $\lambda=227 . \overline{5}$.

In our numerical experiments, we set $y^{*}=0.5384$, but of course operate as if $y^{*}$ is unknown to us. We take $L=20$ measurements of the form (4.8), where the centers $x_{j}$ are chosen at random, applied to the solution $u\left(\cdot, y^{*}\right)$ of (1.6) with $a$ satisfying (4.1)-(4.3) with $s=4$ and $a_{\min }=0.1$. We only see these measurements and not the entire function $u\left(\cdot, y^{*}\right)$.

Our first numerical experiment is to compute the behavior of $\mu_{n}$, the recovery error $\left\|\bar{u}\left(y^{*}\right)-\hat{u}_{n}\right\|_{V}$ and its upper bound $\mu_{n} \epsilon_{n}$, see (4.7), for different choices of $V_{n}$. Here $V_{n}$ is the (adaptive) Taylor reduced space with $n$ terms and $\epsilon_{n}$ is the approximation error computed as discussed in Section 4.1. The values obtained for $n=1,2, \ldots, 20$ when $L=20, s=4$ and $a_{\min }=0.1$ are provided in Figure 3. An important observation from this figure is that increasing the value of $n$ (in order to improve the approximation error) causes $\mu_{n}$ to increase greatly and thereby limits the recovery accuracy. We shall see in the next experiments that this can be circumvented by using piecewise polynomial approximations.

Notice that the dimension $n$ of $V_{n}$ is limited by $n \leq L$ since otherwise $\mu_{n}$ is infinite. This motivates the use of library approximation where the spaces in the library have small dimension $m \leq L$. We do such a numerical experiment using piecewise Taylor polynomial approximation obtained via the adaptive method. We partition $Y$ into 8 cells. This partition corresponds to only subdividing the first coordinate direction $y_{1}$. Each cell gives 


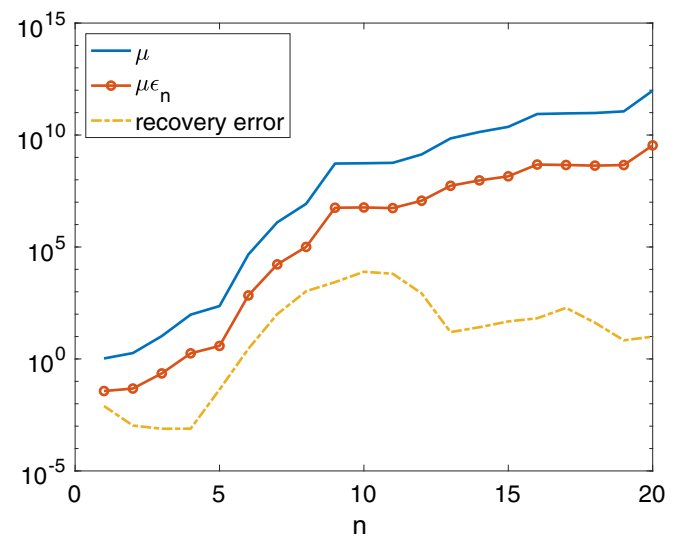

\begin{tabular}{|c|c|c|c|}
\hline$n$ & $\mu_{n}$ & $\mu_{n} \epsilon_{n}$ & $\left\|\bar{u}\left(y^{*}\right)-\hat{u}_{n}\right\|_{V}$ \\
\hline 5 & $2.30600 \times 10^{2}$ & $3.81786 \times 10^{0}$ & $4.30783 \times 10^{-2}$ \\
10 & $5.48266 \times 10^{8}$ & $5.82909 \times 10^{6}$ & $7.82917 \times 10^{3}$ \\
15 & $2.31818 \times 10^{10}$ & $1.43338 \times 10^{8}$ & $4.74617 \times 10^{1}$ \\
\hline
\end{tabular}

FiguRE 3 . The constant $\mu_{n}$, the upper bound $\mu_{n} \epsilon_{n}$ and the recovery error $\left\|\bar{u}\left(y^{*}\right)-\hat{u}_{n}\right\|_{V}$ for the (adaptive) Taylor approximation when $L=20, s=4$ and $a_{\min }=0.1$. Left: graphs for $n=1,2 \ldots, 20$; right: values for $n=5,10,15$.
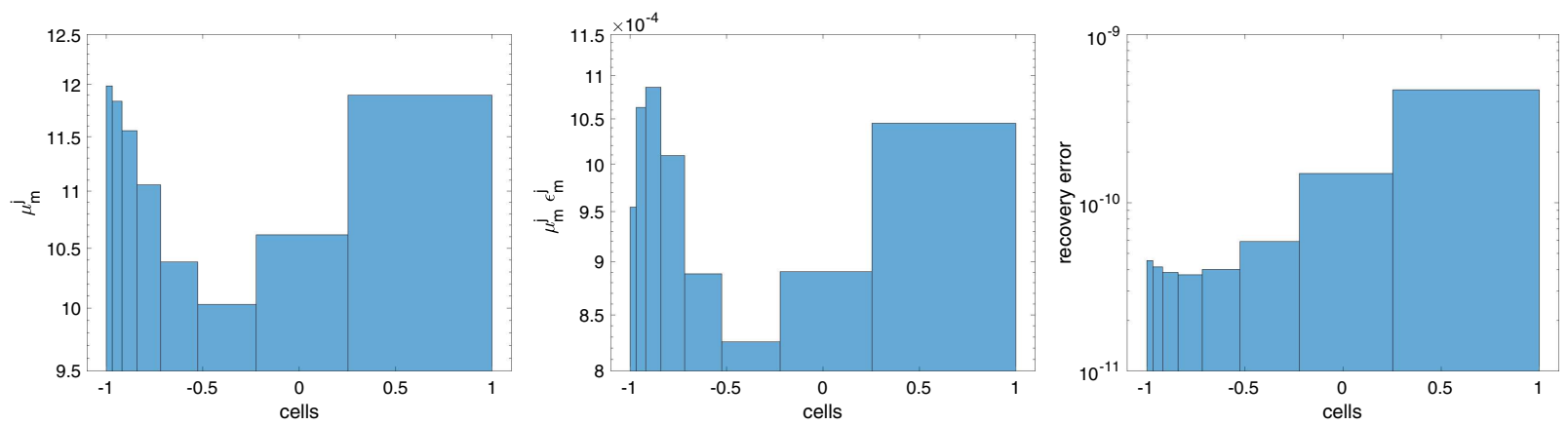

FIgURE 4. Results of the piecewise (adaptive) Taylor polynomial approximation on each cell when $L=20, s=4$ and $a_{\min }=0.1$. Left: the constant $\mu_{m}^{j} ;$ middle: $\mu_{m}^{j} \epsilon_{m}^{j}$; right: recovery error $\left\|\bar{u}\left(y^{*}\right)-\hat{u}_{m}^{j}\right\|_{V}$.

rise to a "local" value of the inf-sup constant $\mu_{m}^{j}:=\mu\left(W, V_{m}^{j}\right), j=1,2, \ldots, 8$, where the $V_{m}^{j}$ 's are the spaces in the library associated with the partition of $Y$. Finally, we use $m=5$ which ensures that the local approximation error satisfies $\epsilon_{m}^{j} \leq \varepsilon=10^{-4}$ for $j=1,2, \ldots, 8$, see Table 1 . Figure 4 gives the value of $\mu_{m}^{j}$, the upper bound $\mu_{m}^{j} \epsilon_{m}^{j}$ and the recovery error $\left\|\bar{u}\left(y^{*}\right)-\hat{u}_{m}^{j}\right\|_{V}, \hat{u}_{m}^{j} \in V_{m}^{j}$, for each cell $j=1,2, \ldots, 8$. Notice that the values of $\mu_{m}^{j}$ do not depend on $y^{*}$.

Also note that the "local" constants $\mu_{m}^{j}$ for the various cells do not exceed 12, while the corresponding $\mu$ was about 230 for the one cell case; see Figure 3-right. Moreover, we observe that for all the cells, the upper bound $\mu_{m}^{j} \epsilon_{m}^{j}$ is smaller than $1.1 \times 10^{-3}$, which ensures that the recovery error (unknown in practice) is less than $1.1 \times 10^{-3}$ for all cells. Note however that we are not providing an algorithm for determining the cell most likely to contain $y^{*}$.

\section{Conclusions}

In this section, we briefly discuss the possible advantages and disadvantages of using nonlinear reduced models in the context of parametric PDEs. We consider only the case of elliptic PDEs (1.6) with affine diffusion 
coefficients (1.7). We suppose that for the given $\left(\psi_{j}\right)_{j \geq 1}$, there is a nondecreasing sequence $\left(\rho_{j}\right)_{j \geq 1}$ with $\rho_{1}>1$ satisfying (3.5). Quantitative theorems for constructing online solvers with performance guarantees are proven using assumptions on the growth of the sequence $\left(\rho_{j}\right)_{j \geq 1}$. The assumption we use here is that the sequence $\left(\rho_{j}^{-1}\right)_{j \geq 1}$ is in $\ell_{q}(\mathbb{N})$ for some $q<2$ (see $\left.[1,4]\right)$. Our discussion is guided by both the theoretical and numerical results of this paper.

\subsection{Offline cost for constructing a solver for linear reduced models}

Let us first consider the case where our interest is constructing an online linear solver for the parametric PDE that performs with a guaranteed approximation error $\varepsilon$. There is a distinction in the offline cost of constructing such a solver, depending on whether it is based on Taylor expansions or on a greedy basis expansion.

When using a Taylor polynomial approximation, we need to find a lower set $\Lambda_{n}^{*}=\Lambda_{n}^{*}(\varepsilon)$ of indices used in the Taylor polynomial expansion (1.3).

We presented two methods for finding such an index set $\Lambda$, which we referred to as the a priori and the adaptive method. The a priori method is numerically cheap since it only requires us to sort the $\rho^{-\nu}$ to identify the largest of these numbers (see [4] for one such sorting algorithm). Once the set $\Lambda_{n}^{*}$ is identified, the Taylor coefficients $\bar{t}_{\nu}$ can be computed recursively with finite element solvers as already discussed. The adaptive method is more expensive as it requires the computation of all $\bar{t}_{\nu}$ in the reduced margin of the adaptively constructed monotone set, while only a few may be included in the set $\Lambda_{n}^{*}$; compare for instance (7.104) and (7.105) in [6]. However, this algorithm is preferred in our numerical experiments presented because it generates sets $\Lambda_{n}^{*}$ with eventually smaller cardinality by assessing precisely the magnitude of $\left\|\bar{t}_{\nu}\right\|_{V}$ instead of using its upper bound $C_{u} \rho^{-\nu}$ (see Lem. 3.14 in [6]).

Consider next the linear reduced model based on the Galerkin projection onto a linear space $V_{n}$ of dimension $n$ constructed by a weak greedy selection of snapshots from the solution manifold. Its main advantage is that $n$ may be much smaller than the number of terms used in the Taylor polynomial approximation (see Fig. 2). Yet, the deficiencies in such greedy algorithms are that the offline cost for the selection of the greedy basis using an $\varepsilon$-net training set grows like $\mathcal{O}\left(\varepsilon^{-c / r} e^{C \varepsilon^{-1 / r}}\right)$ (see for instance (8.89) together with (8.108) from [6]) which may be prohibitive for small $\varepsilon$. This was one of the main motivations for using nonlinear models in place of linear models.

\subsection{Offline cost for constructing a solver using nonlinear reduced models}

We discuss next the offline cost in the construction of nonlinear reduced models based on piecewise Taylor polynomials. We have given a priori recipes for the tensor product partitioning of $Y$ into cells $Q$ based on the knowledge of the sequence $\left(\rho_{j}\right)_{j \geq 1}$, and thus the main issue is building the appropriate basis for each cell $Q$ of this partition. This requires the computation of the finite element approximation of the appropriate Taylor coefficients on each cell. Note that these computations can be done in parallel. The total cost of this offline construction is governed by $N$, the total number of cells in the partition, and $m$, the number of terms used on each cell. In our numerical examples, these constructions were not an issue because the number of cells $N$ was reasonable for moderate values of $m$.

We have given a priori bounds on the number of cells needed for the partition in Section 3.2. Recall that if we are in a situation where linear methods (such as polynomial or greedy) give an approximation rate $\mathrm{Mn}^{-r}$, then we can guarantee an approximation error $\varepsilon=n^{-r}$ by using piecewise polynomials with $m$ terms and $N \leq e^{C(n / m)^{\alpha}}$ cells, at least in the special case discussed in Section 3.3. If we assume the cost of creating a polynomial approximation with $m$ terms scales like $e^{c m}$, which we know is the case for greedy constructions, then the cost for constructing the piecewise polynomial is bounded by $e^{C(n / m)^{\alpha}+c m}$. By choosing $m<n$ appropriately, this is always less than the cost of the approximation without partitioning, which is $e^{C n}$. For example, if $\alpha=1$ then we could choose $m=\sqrt{n}$ and get the total piecewise polynomial cost to be $e^{C \sqrt{n}}$ as compared with the $e^{C n}$ if we do not partition. In our numerical examples, we have seen that the a priori bounds on the number of 
cells is quite pessimistic, and we actually get better performance than that predicted by the a priori estimates for the number of cells.

\subsection{Online cost for constructing the approximate solution for linear reduced models}

If we use a linear reduced model based on Taylor polynomials, then once the index set $\Lambda$ is found and the Taylor coefficients $\bar{t}_{\nu}, \nu \in \Lambda$, are computed, the reduced model is

$$
\bar{u}(y)=\sum_{\nu \in \Lambda} \bar{t}_{\nu} y^{\nu} .
$$

Thus, given a parameter query, the online cost for the evaluation of $\bar{u}(y)$ is trivial.

If we use a greedily generated linear space $V_{n}$ of dimension $n$, there are additional online costs. Given a parameter query $y$ one must find the Galerkin projection of $u(y)$ onto $V_{n}$. This entails the inversion of an $n \times n$ dense matrix where the matrix depends on $y$. In certain cases, such as when the diffusion coefficient is affine, this can be somewhat mitigated by precomputing certain matrices (see the discussion in [6]). Therefore, there is a balancing between having a smaller dimensional reduced model (when compared with the polynomial case) and the additional cost of matrix inversion in an online solver.

Notice also that the accuracy of the online performance for reduced models using Taylor polynomials can be improved by using a Galerkin projection onto the polynomial space in place of the plug in formula. However, this projection would also involve an expensive matrix inversion.

\subsection{Online cost for constructing the approximate solution for nonlinear reduced models}

Building an online solver based on piecewise Taylor polynomial approximations proceeds by building a linear solver for each cell of the partition. An additional step is required to determine which space from the library of spaces should be used for the query $y$. This only requires the identification of the cell which contains $y$, and is easily determined from the knowledge of the partition since the cells are hyperrectangles.

\subsection{Storage costs}

The storage cost for the online solver is dominated by the storage of the basis functions. They are typically large vectors depending on $\varepsilon, D$ and $f$ in (1.6). We observe from our numerical experiments that the storage cost is higher for linear reduced models using Taylor polynomials compared to the greedy reduced basis algorithm; see Figure 2. Moreover, the costs for Taylor polynomial reduced models and piecewise Taylor polynomial reduced models are quite comparable. For example, Table 2 shows that for a target accuracy $\varepsilon=10^{-4}$ and $s=3$, $a_{\text {min }}=0.01$, the linear reduced model uses 614 basis functions $\bar{t}_{\nu}$ while the piecewise Taylor construction has 48 cells with $m=9$ terms on each cell, and hence requires the storage of 432 vectors.

\subsection{Summary}

Linear reduced models based on Taylor polynomials have several advantages, including simple identification of the set $\Lambda$ with no need for optimization or search algorithms, and fast computation of the online solver $\bar{u}(y)$. The deficiency in such constructions is that to reach a small target accuracy $\varepsilon$ the dimension $m=\# \Lambda$ may be very large and thus affect the offline construction. A large value of $m$ would also lead to large storage costs.

The advantage of a greedily chosen linear reduced model is that the dimension required for it to reach a target accuracy is typically much smaller than what is required when using Taylor polynomials. However, there is typically a larger offline cost to construct the greedy basis when the required dimension is large, and the costs of executing an online solver are higher. On the other hand, because the dimension of the greedy space is small, there is a savings in storage.

These considerations lead us to consider replacing the linear reduced model by nonlinear models, and in this work we considered nonlinear reduced models based on piecewise polynomial approximation. These piecewise polynomial models have the advantage of being able to achieve a better accuracy than linear reduced models 
while still taking $m$ small, provided that the number of cells $N$ in the piecewise construction is moderate. In this paper, we have given both a priori bounds on the necessary size of $N$ as well as numerical bounds. Both bounds show the advantage of this approach. The potential deficiency of this approach is a large storage cost if $N$ is large. Our numerical examples suggest that $N$ is considerably smaller than the a priori bounds, thereby making this a viable approach when the desired accuracy $\varepsilon$ is small.

Acknowledgements. The authors would like to thank Matthew Hielsberg for his efforts in implementing the numerical algorithms and producing the charts and figures. This research was supported by the NSF grants DMS 1817691 (AB), DMS 1817603 (RD-GP), ONR grant N00014-17-1-2908 (RD); DG was supported by the Swiss National Science Foundation grant P2ELP2-175056 and IAMCS at TAMU, and PJ was supported by an NSF Fellowship DMS 17-04121. A portion of this research was completed while AC, RD (Simon Fellow), DG, and PJ were supported as visitors of the Isaac Newton Institute at Cambridge University.

\section{REFERENCES}

[1] M. Bachmayr, A. Cohen and G. Migliorati, Sparse polynomial approximation of parametric elliptic PDEs. Part I: affine coefficients. ESAIM:M2AN 51 (2017) 321-339.

[2] P. Binev, A. Cohen, W. Dahmen, R. DeVore, G. Petrova and P. Wojtaszczyk, Convergence rates for greedy algorithms in reduced basis methods. SIAM J. Math. Anal. 43 (2011) 1457-1472.

[3] P. Binev, A. Cohen, W. Dahmen, R. DeVore, G. Petrova and P. Wojtaszczyk, Data assimilation in reduced modeling. SIAM/ASA J. Uncertainty Quantification 5 (2017) 1-29.

[4] A. Bonito, R. DeVore, D. Guignard, P. Jantsch and G. Petrova, Polynomial approximation of anisotropic analytic functions of several variables. Constructive Approximation (2020) 1-30.

[5] A. Chkifa, A. Cohen, R. DeVore and C. Schwab, Sparse adaptive Taylor approximation algorithms for parametric and stochastic elliptic PDEs. ESAIM: M2AN 47 (2013) 253-280.

[6] A. Cohen and R. DeVore, Approximation of high-dimensional parametric PDEs. Acta Numer. 24 (2015) 1-159.

[7] A. Cohen and G. Migliorati, Multivariate approximation in downward closed polynomial spaces. In: Contemporary Computational Mathematics - A Celebration of the 80th Birthday of Ian Sloan. Springer (2018) 233-282.

[8] A. Cohen, W. Dahmnen, R. DeVore and J. Nichols, Reduced basis greedy selection using random training sets. ESAIM:M2AN 54 (2020) 1509-1524.

[9] R. DeVore, Nonlinear approximation. Acta Numer. 7 (1998) 51-150.

[10] R. DeVore, G. Petrova and P. Wojtaszczyk, Greedy algorithms for reduced bases in Banach spaces. Constr. Approximation 37 (2013) 455-466.

[11] J.L. Eftang, A.T. Patera and E.M. Rønquist, An "hp" certified reduced basis method for parametrized elliptic partial differential equations. SIAM J. Sci. Comput. 32 (2010) 3170-3200.

[12] Y. Maday and B. Stamm, Locally adaptive greedy approximations for anisotropic parameter reduced basis spaces. SIAM J. Sci. Comput. 35 (2013) A2417-A2441.

[13] Y. Maday, A.T. Patera, J. Penn and M. Yano, A parameterized-background data-weak approach to variational data assimilation: formulation, analysis, and application to acoustics. Int. J. Numer. Meth. Eng. 102 (2014) 933-965.

[14] G. Pisier, The Volume of Convex Bodies and Banach Space Geometry. Cambridge University Press 94 (1999).

[15] V. Temlykov, Nonlinear Kolmogorov widths. Math. Notes 63 (1998) 785-795.

[16] Z. Zou, D. Kouri and W. Aquino, An adaptive local reduced basis method for solving PDEs with uncertain inputs and evaluating risk. Comput. Methods Appl. Mech. Eng. 345 (2019) 302-322. 